\title{
Energy Saving in Carrier-Grade Networks: A Survey
}

\author{
Rihab Maaloul \\ National School of Engineering, \\ LETI laboratory, \\ Sfax, Tunisia \\ rihab.maaloul.abid@gmail.com
}

\author{
Lamia Chaari \\ National School of Engineering, \\ LETI laboratory, \\ Sfax, Tunisia \\ Lamia.Chaari@enis.rnu.tn
}

\author{
Bernard Cousin \\ University of Rennes 1 \\ IRISA, \\ Rennes, France \\ $<$ first-name>.<name>@irisa.fr
}

\begin{abstract}
Summary - Energy consumption of large-scale networks has become a primary concern in a society increasingly dependent on information technology. Novel solutions that contribute to achieving energy savings in wired networks have been proposed to mitigate ongoing and alarming climate change and global warming. A detailed survey of relevant power-saving approaches in wired networks is presented here. We give a special focus on carrier-grade networks. At first we perform a comprehensive study of communication infrastructures regarding energy saving. Then, we highlight key issues to enable green networks, ranging from network design to network operation. After that, we present the major contributors to power consumption in wireline networks. Afterwards, we survey, classify, and compare the main energyaware methods and mechanisms that are the most appropriate for improving the energy efficiency of carrier-grade networks.
\end{abstract}

Keywords-Green networking; wired networks; energy consumption in network devices; energy-aware traffic engineering; carrier grade networks

\section{INTRODUCTION}

Reducing electricity bills and energy consumption has become a crucial goal for all industries, including the Information and Communication Technology (ICT) sector, as it is rapidly becoming an important play-actor in daily life $[1,2]$. The alarming figures reported by worldwide energy consumption have pushed telecom operators to rethink their network policy [3]. Nowadays, the function of the ICT is progressed by addressing energy awareness in all phases of production and service delivery. Energy-aware studies in communication networks, especially with respect to the environmental conditions, are commonly referred to as green networking.

As the traffic demand continues to grow, it requires additional network resources with higher capacity and faster processing speeds. Moreover, the improvements in network infrastructure drive the quest for green networking. Particularly, transport and carrier grade networks represent permanent and extensive resources of power consumers. For instance, data center operators require a considerable amount of power to operate server stacks, storage equipment, cooling equipment, operation room and so on. Green networking has two main reasons [4]:

1) The enviromental reason: most energy consumption is accompanied by non-negligible GHG (Green House Gas) emission that has harmful consequences on climate. In addition, a decrease in GHG emission volume between 15-
$30 \%$ is required before 2020 to keep the global temperature increase below $2^{\circ} \mathrm{C}$ [5].

A large set of telecom operators and Internet Service Providers (ISPs) consider GHG reduction and its ecological impacts. In fact, the volume of carbon dioxide emissions produced by the ICT sector alone is estimated to be over $2 \%$ of the total world carbon footprint in 2020 [6]. In 2007, this $2 \%$ was equivalent to 830 million metric tonnes of carbon dioxide [7] and it would be about 1100 million tonnes by $2020[8,9]$.

Statistical reports provided by certain telecom operators state the overall amount of their power requirements and the related carbon footprint [10-12]. All of these studies show that ICT energy consumption represents an important carbon dioxide emission and will increase rapidly if no green technique is adopted. It might account for more than 35.8 TWh by 2020 [196][197].

2) The economic reason: the rapid increasing of CAPEX (Capital Expenditure) and OPEX (Operational Expenditure) represents a major economical concern. CAPEX is related to network infrastructure establishment cost, whereas OPEX is related to network operation and administration. Energy costs have been investigated by the operators and their financial damage has been put in perspective. Figure 1 shows the constantly rising energy costs. Moreover, [13] anticipates that a one-third reduction of carbon footprint emissions could create an economical benefit greater than the investments required to attain this goal. Table I presents the cost of energy devoted for network devices The estimation of energy consumption is based on the primary/seminal study done by [14], which states the annual electricity consumed by networking devices in the U.S. was $6.06 \mathrm{TWh}$, which costs USD 1 billion per year and it is equivalent to one nuclear reactor.

As a result of these two reasons, international projects and research bodies have focused on developing green network infrastructures. We show here the key enablers to understand the source of energy waste and by what means energy could be saved. Also we present the most relevant achievements that allow a better ratio of performance to energy consumption in wired networks. The emergence of a multitude of approaches and mechanisms on power saving necessitates a study and an analysis of these different approaches in order to identify and classify the potential mechanisms for different scenarios and network domains. 


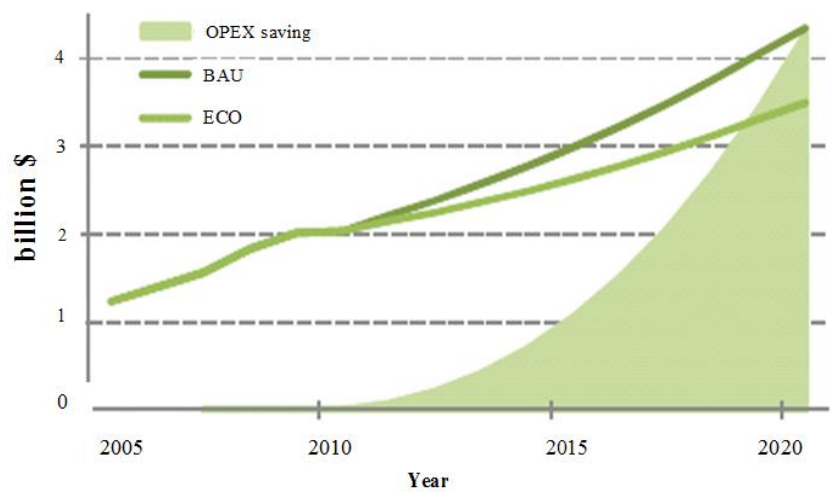

Figure 1. Estimated OPEX for the European telcos' network infrastructures in the "Business-As-Usual" (BAU) and in the Eco sustainable (ECO) scenarios, and cumulative savings between the two scenarios [4].

We place specific emphasis on energy-saving studies dedicated to carrier-grade transport networks $[15,16]$. These networks are energy-hungry infrastructures; they run largescale systems to deliver internet services. We choose correspondingly to overview approaches that could be helpful and adapted to carrier-grade transport networks. Carrier grade means extremely high reliability and refers to the capability to support thousands if not millions of subscribers [17]. To the best of our knowledge, it doesn't exist any review focalized on carrier-grade networks. A carrier-grade network is not a single technology, but rather a collection of different technologies. A set of functionalities and requirements must be defined in carrier-grade communication: (1) Scalability; (2) Resilience; (3) Quality of service; and (4) Service management. In networks that involve carrier-grade requirements, power saving often induces the reduction of network redundancy or network performance. For instance, in order to meet the resiliency and quality of service requirements, the network should provide fast fault recovery (under $50 \mathrm{~ms}$ ) through a number of duplicated resources that are not used frequently. Considering the performance trade-off versus power saving, designing efficient power-saving strategies is a real challenge. Nevertheless, the green communications and networking fields are still in their early stages; yet they have already spurred a considerable number of interesting works, which are surveyed and analysed here.

The remainder of this article is organised as follows. Section II provides an understanding of how different parts in the network infrastructure contribute to power consumption. Section III explains the inevitable synergy of several paradigms that reduce energy consumption. Section IV presents the concept of energy profiles for routing equipment. Section $\mathrm{V}$ focuses on the frequent network architectures used in carrier-grade networks. Section VI proposes a classification with an analysis of the relevant approaches in the field of green networking. Afterward, the main issues of energy-efficient and operational carrier-grade networks are presented in Section VII. Finally, Section VIII concludes the study.

\section{PRINCIPAL CONTRIBUTORS TO NETWORK ENERGY CONSUMPTION}

In order to gain a complete view of the principal contributors to energy consumption, it is crucial to consider the communication networks globally from the user level to the transport level, as shown in Figure 2. We identify three key contributors that consume energy within the overall network infrastructure: network devices, network architecture, and delivered services.

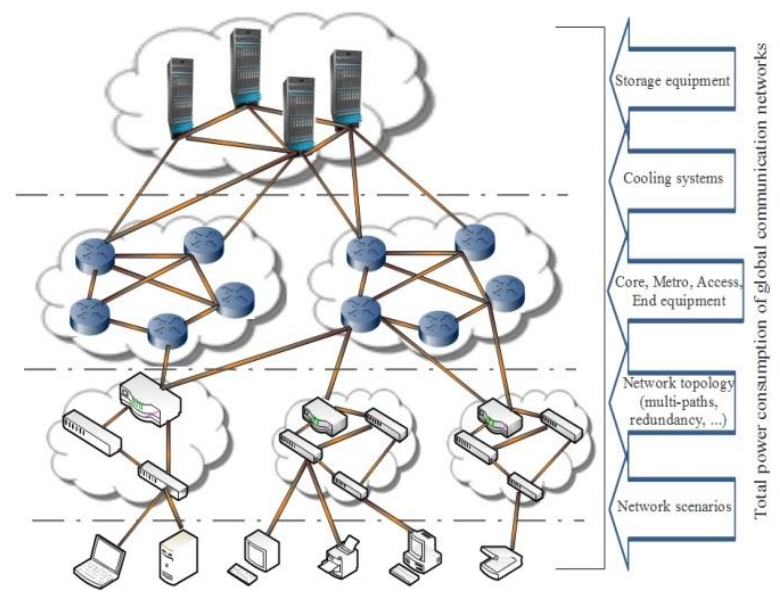

Figure 2. Overall network energy consumption

\section{A. Network devices}

The most important contributor to the power expenditure of network systems are the physical networking devices. This includes elements in different network domains: core, metro, and access networks. Several strategies have been proposed for the energy management of networking devices, ([18] among others).

TABLE I. POWER CONSUMPTION SUMMARY FOR NETWORK DEVICES [18]

\begin{tabular}{|c|c|c|c|c|c|c|}
\hline $\begin{array}{c}\text { Device } \\
\text { category }\end{array}$ & $\begin{array}{c}\text { Rated } \\
\text { Max } \\
\text { Power } \\
\text { (in \%) }\end{array}$ & $\begin{array}{c}\text { Measured } \\
\text { Max } \\
\text { Power } \\
(\mathrm{M})^{\mathrm{a}}\end{array}$ & $\begin{array}{c}\text { Measured } \\
\text { Idle Power }\end{array}$ & $\begin{array}{c}\text { EPI }^{\mathrm{b}} \\
\text { (in \%) }\end{array}$ & $\begin{array}{c}\text { Aggregate } \\
\text { bandwidth } \\
\text { in Mbit/s }\end{array}$ & $\begin{array}{c}\text { mW } \\
\text { /Mbit/s }^{\mathrm{c}}\end{array}$ \\
\hline $\begin{array}{c}10 / 100 \\
\text { Hub }\end{array}$ & 35 & 12.8 & 11.7 & 8.59 & 1200 & 10.7 \\
\hline $\begin{array}{c}\text { Edge } \\
\text { LAN } \\
\text { switch }\end{array}$ & $759^{\mathrm{d}}$ & 198 & 150 & 24.2 & 48000 & 4.1 \\
\hline $\begin{array}{c}\text { Edge } \\
\text { LAN } \\
\text { switch }\end{array}$ & $857^{\mathrm{e}}$ & 175 & 133.5 & 23.7 & 48000 & 3.7 \\
\hline $\begin{array}{c}\text { Edge } \\
\text { LAN } \\
\text { switch }\end{array}$ & 300 & 102 & 76.4 & 25.1 & 48000 & 2.1 \\
\hline $\begin{array}{c}\text { Core } \\
\text { switch }\end{array}$ & 3000 & 656 & 555 & 15.4 & 48000 & 13.7 \\
\hline $\begin{array}{c}\text { Edge } \\
\text { router }\end{array}$ & 300 & 210 & 168.5 & 19.8 & 24000 & 8.75 \\
\hline
\end{tabular}

${ }^{a} M$ is the amount of the power consumed in $W$,

${ }^{b}$ Energy Proportionality Index 
${ }^{c}$ Measured max power in mW / Aggregate bandwidth in Mbit/s. This term is equivalent to Joules per bit.

${ }^{d}$ including $400 \mathrm{~W}$ for PoE (P over Ethernet )

${ }^{e}$ including $400 \mathrm{~W}$ for PoE .

Each type of network device (hubs, routers, switches ...) has its own architecture and functionalities. Hence, each network device presents a power consumption that is influenced by many factors such as manufacturer type, number of active ports, number of line cards, traffic characteristics, and used protocols. Since there is no standard used in powerline measurement of network devices, some benchmarks are used as reference to characterize the power consumption. Indeed, various workers have proposed models to describe the energy consumed by network devices such as hubs, switches, routers, and other network devices, starting from the pioneering work of [14] and following works such as [1821]. Table I lists the power consumption of the main network devices, as shown in [18]. We observe that almost every specified device demonstrates non-proportional energy consumption behaviour, as shown by the EPI values. Thus we observe significant independency between the energy consumed and the traffic throughput. However, relying only on the power consumed at the maximum rate reported by data sheets can overestimate the current power consumption.

Other studies [22-24] focus on minimizing the power dissipation of specific components such as Network Interface Card (NIC), hard disks, and CPUs. Thus, [25] measure the power consumed by the main components of a typical rack server (Table III).

European Union (EU) has published power consumption guidelines in different updated version of conduct code on energy consumption of broadband equipment. In this respect, we reproduce in Table II the power values for WAN components interfaces [119].
TABLE II. POWER CONSUMPTION FOR MAJOR COMPONENTS OF A TYPICAL SERVER[25]

\begin{tabular}{cccc}
\hline Component & $\begin{array}{c}\text { Peack power } \\
(\mathrm{W})\end{array}$ & Count & Total (W) \\
\hline CPU & 100 & 2 & 200 \\
Memory & 20 & 4 & 80 \\
Disk & 10 & 1 & 10 \\
Motherboard & 40 & 1 & 40 \\
Fan & 30 & 1 & 30 \\
\hline System total & & & 360 \\
\hline
\end{tabular}

TABLE III

POWER CONSUMPTION FOR WAN INTERFACES [119]

\begin{tabular}{|c|c|c|}
\hline \multirow{2}{*}{ Component } & \multicolumn{2}{|c|}{$\mathbf{2 0 1 3 - 2 0 1 4}$} \\
\cline { 2 - 3 } & $\begin{array}{c}\text { Idle-State } \\
\text { (W) }\end{array}$ & $\begin{array}{c}\text { On-State } \\
(\mathbf{W})\end{array}$ \\
\hline Fast Ethernet WAN & 2.0 & 3.0 \\
\hline Gigabit Ethernet WAN & 2.5 & 5.0 \\
\hline FibrePtPFast Ethernet WAN & 2.9 & 5.0 \\
\hline Fibre PtPGigabit Ethernet WAN & 3.2 & 5.6 \\
\hline 10/1G-EPON & 4.8 & 6.2 \\
\hline 10/10G-EPON & 5.3 & 7.7 \\
\hline XG-PON1 & 4.8 & 6.5 \\
\hline $\begin{array}{c}\text { Gigabit Passive Optical Network } \\
\text { (GPON) }\end{array}$ & 3.5 & 5.0 \\
\hline $\begin{array}{c}\text { Ethernet Passive Optical Network } \\
\text { (EPON) }\end{array}$ & 3.5 & 4.7 \\
\hline
\end{tabular}

Figure 3 shows the contribution of different types of network device to the worldwide energy consumption according to the analysis of Lawrence Berkeley National Laboratory (LBNL) campus [26] in 2009. These figures demonstrate that network switching and premises equipment are the largest categories, for about $70 \%$, of the overall energy use.

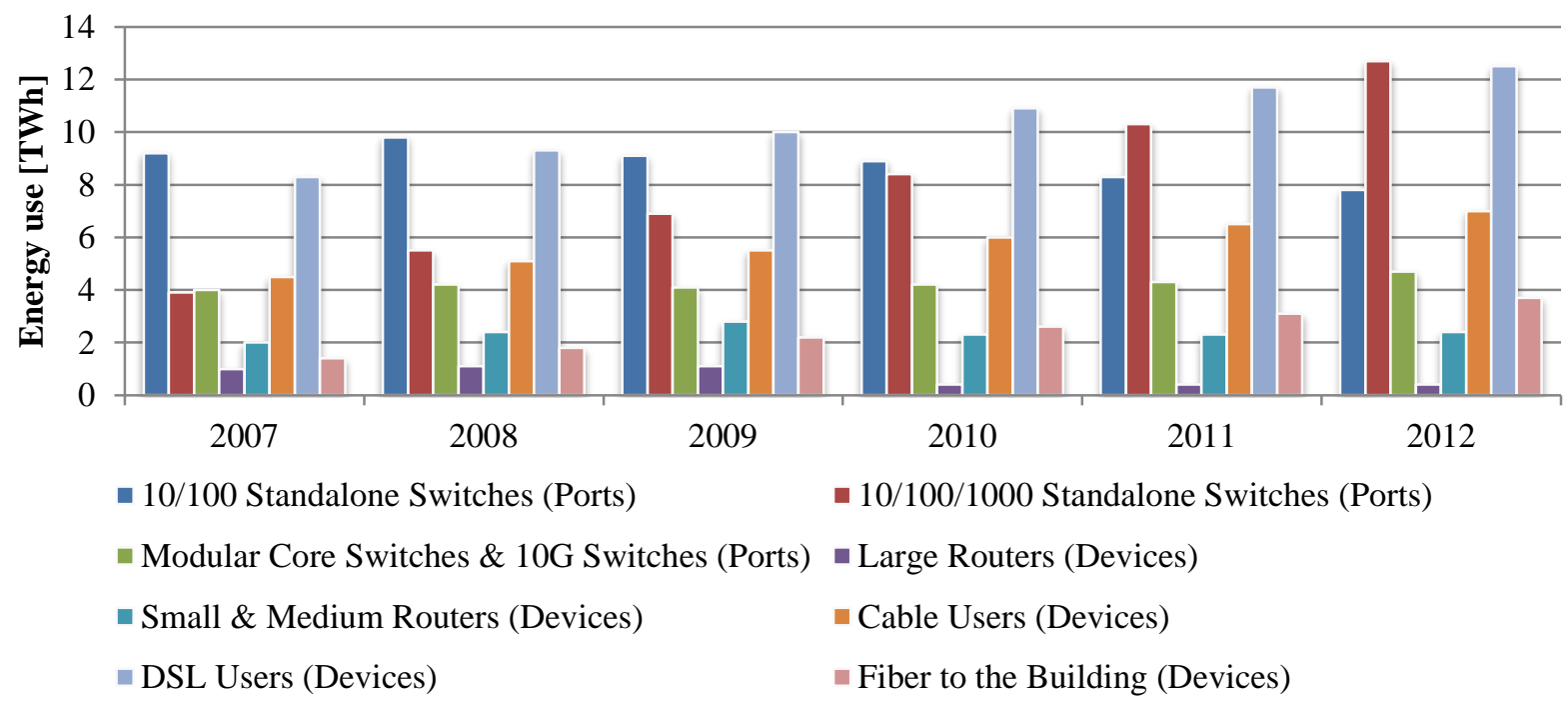

Figure 3. Energy use of netowrk equipment 
Due to the technological advances in the ICT field, there is an important necessity for a permanent evaluation of the energy consumed by network devices. Such an evaluation is achieved by the cooperation of network manufacturers, ISPs, standard organizations, and national regulators [27].

\section{B. Network architecture}

The network architecture is the design of the telecom network that specifies the network's physical elements and their operational configuration. The network architecture is typically split into three network domains: core, metro, and access networks. In these different domains, the equipment involved, their objectives, their expected performance, and their power consumption levels differ. According to [28], Telecom Italia was the second largest consumer of energy in Italy, consuming more than $2 \mathrm{TWh}$ per year. Table IV shows overall consumption foreseen for each network domain of Telecom Italia for the years 2015-2020.

TABLE IV. 2015-2020 NETWORK FORECAST/DEVICE DENSITY AND ENERGY REQUIREMENTS IN THE BUSINESS-AS-USUAL (BAU). EXAMPLE BASED ON THE ITALIAN NETWORK[28] .

\begin{tabular}{|c|c|c|c|}
\hline & $\begin{array}{c}\text { Power } \\
\text { consumption } \\
{[\mathrm{W}]}\end{array}$ & $\begin{array}{c}\text { Number of } \\
\text { devices } \\
{[\#]}\end{array}$ & $\begin{array}{c}\text { Overall } \\
\text { consumption } \\
{[\mathrm{GWh} / \text { year }]}\end{array}$ \\
\hline Home & 10 & $17,500,000$ & 1,533 \\
\hline Access & 1,280 & 27,344 & 307 \\
\hline Metro & 6,000 & 1,750 & 92 \\
\hline Core & 10,000 & 175 & 15 \\
\hline \multicolumn{3}{|c|}{ Overall network consumption 1,947} \\
\hline
\end{tabular}

- Core network: often referred to as the backbone network or as the long-haul infrastructure, which interconnects large cities over continental and even intercontinental distances [29]. The core network is based on a mesh interconnection pattern and carries a large volume of traffic. In the backbone network, pairs of routers are typically connected by multiple physical cables that form one logical bundled link [30] that participates in intra-domain routing protocol. Link bundles are accustomed because when capacity is upgraded, new links are joined beside the existing ones, rather than replacing the existing equipment with a higher capacity link. For example, a $40 \mathrm{Gbit} / \mathrm{s}$ bundled link can involve four OC-192 cables with $10 \mathrm{Gbit} / \mathrm{s}$ of capacity for each cable.

In 2009, [31] suggested an increase of core network consumption: by 2017, the power consumption of the core network will be equal to that of network access. Furthermore, this study predicts a staggering increase of $300 \%$ in power consumption of the core domain in the coming decade.

- Metro network: this is the domain of the telecom network that typically covers metropolitan regions. It aggregates the highly fluctuating traffic of residential subscribers from the end user to the core network serving as an interface between the access and the core. Different networking technologies have been deployed in diverse metro regions across the world. Today's dominant metropolitan area networks (MAN) are: SONET (Synchronous optical Networking), Optical WDM ring, and Metro Ethernet Hierarchy. Nowadays, ISPs tend to offer Ethernet in MAN networks, which are arguably more flexible, scalable, and cost-effective compared to legacy SONET architecture [32]. Its basic components are edge routers, broadband network gateways, and Ethernet switches.

- Access network: this part represents the end users connected to POPs (Point of Presence) via a multitude of physical media (e.g. optical, DSL, wireless). It is a major consumer of energy because it comprises a huge number of active elements [33]. There are several different access technologies that can be roughly classified into two main categories: wired and wireless. A detailed analysis of the energy consumption for the main used access technologies was given in [34], [71], and [120].

\section{Services}

Power consumption is influenced by the nature of the services being provided. In other words, the system activity and the usage of network resources deployed to deliver network services. Indeed, the greatest amount of Internet traffic arises from a wide range of web-based services and applications available to end users via the Internet [35], such as cloud services, content delivery and storage as a service. For instance, content services involve servers that store up the data/content and control access to it. The ability to run any of these services assumes that the network system has sufficient power to perform the task.

The network system offers the following types of services:

- Shared services: such as Network File System (NFS), web browsing and email. These services can be oversubscribed in that many users may share the offered bandwidth without observing any degradation in the quality of their service.

- Dedicated hosting services imposing different levels of quality of service: a dedicated network resource for each service has to be supplied through the access and backhaul network to the hosting servers.

Cloud services: cloud computing is expected to be the future internet service model by offering network-based rather than desktop-based applications [36]. Clouds exploit a significant amount of equipment and management techniques to allow customers to share a large pool of software, storage, platforms, and computational resources [37]. The most popular cloud based services are content delivery, Storage as a Service (StaaS) and virtual machines-based applications. Also, these services consume various levels of energy according to their proprieties, i.e. complexity, heterogeneity and large scale. 
The key points for greening carrier-grade networks are the green operations of network infrastructure, the delivery of services through energy-efficient equipment and implemented power management, as outlined in the next section.

\section{BUILDING A GREEN NETWORK}

Making a network operate in green way compels the identification of several issues using energy efficiency as the primary goal. In order to obtain a green network, at least four key issues should be adopted, as presented in Figure 4.

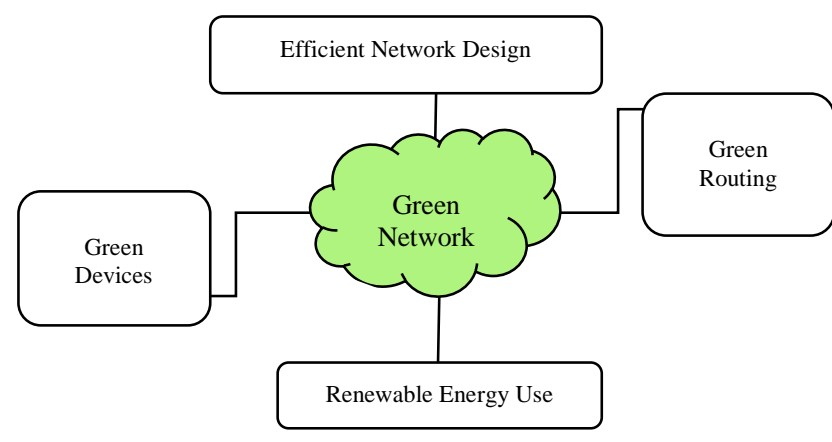

Figure 4. Key issues for greening networks

The green devices issue seeks to build a new generation of energy-efficient devices. In this respect, over the next few years, industry has developed energy-efficient and lowcarbon technologies. In order to accomplish this objective, 10 key actions should be realised that are outlined in [38]. [39] proposes to replace the electronic circuits within routers by photonic circuits. They could reach over $10 \mathrm{Tbit} / \mathrm{s}$ of attainable speed versus $100 \mathrm{Gbit} / \mathrm{s}$ attained by electronics. However, the power consumption of photonic signal processing technologies is not feasible to be adopted. The same workers, in one of their next works [40], presented a perspective on device power consumption. They claim that network devices working in the different parts of the network play a crucial role because the main power consumption in networks comes from their operational power exigencies and density.

The GreenTouch[121] and ECONET[122,123] initiatives are the most relevant green networking projects appeared by the year 2010. GreenTouch focused on all-optical networking systems, while the ECONET project has contributed to speed-up the reduction of energy consumption in silicon elements of network devices, as well as in copper-based access technologies (namely, VDSL), which both are expected to be used and deployed in telco networks up to the next 15 years.

The efficient network design issue aims at dimensioning the network architecture and organising the devices in such a way that they consume a lower amount of energy. The European Commission realised the significance of this issue, which appeared in its $7^{\text {th }}$ Framework Program FP7 [41]. This later activated the TREND project (Toward Really Energy-
Efficient Network Design), which has certain pertinent achievements [42]:

- Collection of comparable data to assess the power consumption of terminals, devices and infrastructures, and the identification of power consumption trends in networking.

- Identification of energy-friendly devices, technologies, algorithms, protocols and architectures, and the investigation of how they can be introduced into operational networks.

- Definition of new energy-aware network design criteria.

- Experiments that prove the effectiveness of the proposed approaches.

- Identification of a roadmap for energy-efficient networking.

Green routing issues aim at introducing energy-aware mechanisms inside routing protocols, which would be able to manage the power state of network resources dynamically. In this regard, numerous studies have proposed several energy-efficient algorithms to route the traffic [4347]. These works focus on routing path selection subject to minimising the energy consumption, whether by powering off devices (or part of them) or by routing the traffic through energy-efficient paths that are weighted according to their energy impact [48].

On the other hand, [49] validated that geographical delocalisation is a promising approach to reduce the cost of electricity related to the routing. In fact, large companies like Amazon have systems that are geographically distributed where electricity has a lower cost. However, this technique is helpful from an economical point of view, but does not reduce the power consumption.

Renewable energy use aims at exploiting renewable resources such as the Sun, wind, and water, hence reducing expenditure as well as the emission of a carbon footprint.

In this respect, renewable energy utilisation makes use of several concepts such as the option to choose a greener resource, electricity price-based, energy resource availability and the localisation of renewable energy generators for smart grid communications and renewable electricity use [50-52].

Moreover, a number of popular ICT companies are becoming dynamic supervisors in their electricity use. Table $\mathrm{V}$ indicates information on renewable electricity and carbon footprint reduction goals identified by ICT organisations [53].

TABLE V. RENEWABLE ELECTRICITY AND CARBON REDUCTION GOALS FOR LEADING ICT ORGANISATIONS[53].

\begin{tabular}{|c|c|c|c|c|}
\hline Company & $\begin{array}{c}\text { Renewable } \\
\text { Energy Goals }\end{array}$ & $\begin{array}{c}\text { Renewable } \\
\text { Energy } \\
\text { Goal year }\end{array}$ & $\begin{array}{c}\text { Carbon } \\
\text { Reduction } \\
\text { Goal }\end{array}$ & $\begin{array}{c}\text { Carbon } \\
\text { Reduction } \\
\text { Goal Year }\end{array}$ \\
\hline Amazon & $100 \%$ & Long-Term & $\begin{array}{c}\text { None } \\
\text { Specified }\end{array}$ & $\begin{array}{c}\text { None } \\
\text { Specified }\end{array}$ \\
\hline Apple & $100 \%$ & Long-Term & $\begin{array}{c}\text { None } \\
\text { Specified }\end{array}$ & $\begin{array}{c}\text { None } \\
\text { Specified }\end{array}$ \\
\hline
\end{tabular}




\begin{tabular}{|c|c|c|c|c|}
\hline Cisco & $25 \%$ & $\begin{array}{c}\text { Annual } \\
\text { Goal: } 2013- \\
2017^{\mathrm{a}}\end{array}$ & $40 \%$ & 2017 \\
\hline Dell & $50 \%$ & 2020 & $50 \%$ & 2020 \\
\hline eBay & $8 \%$ & 2015 & $\begin{array}{c}10 \% \text { reduction } \\
\text { in carbon per } \\
\text { transaction in } \\
2013\end{array}$ & 2013 \\
\hline Facebook & $100 \%$ & Long-Term & $\begin{array}{c}\text { None } \\
\text { Specified }\end{array}$ & $\begin{array}{c}\text { None } \\
\text { Specified }\end{array}$ \\
\hline Google & $100 \%$ & Long-Term & $\begin{array}{l}\text { Carbon } \\
\text { Neutral }\end{array}$ & Ongoing \\
\hline IBM $^{\mathrm{b}}$ & $\begin{array}{c}\text { None } \\
\text { Specified }\end{array}$ & $\begin{array}{c}\text { None } \\
\text { Specified }\end{array}$ & $\begin{array}{c}\text { Third- } \\
\text { Generation } \\
\text { GHG Goal } \\
\text { Upcoming }\end{array}$ & Upcoming \\
\hline Intel & $\begin{array}{c}\text { None } \\
\text { Specified }\end{array}$ & $\begin{array}{c}\text { None } \\
\text { Specified }\end{array}$ & $\begin{array}{l}10 \% \text { reduction } \\
\text { per chip in } \\
\text { GHG direct } \\
\text { emissions }\end{array}$ & 2020 \\
\hline Microsoft & $100 \%$ & 2014 & $\begin{array}{l}\text { Carbon } \\
\text { Neutral } \\
\end{array}$ & 2014 \\
\hline Rackspace & $\begin{array}{l}5 \% \text { increase } \\
\text { annually until } \\
\text { goal attained } \\
(35 \% \text { in } 2013)\end{array}$ & $\begin{array}{l}2026 \text { (based } \\
\text { on } 5 \% \\
\text { annual } \\
\text { increase } \\
\text { from } 35 \% \text { in } \\
\text { 2013) }\end{array}$ & $\begin{array}{c}\text { None } \\
\text { Specified }\end{array}$ & $\begin{array}{c}\text { None } \\
\text { Specified }\end{array}$ \\
\hline Sprint & $10 \%$ & 2017 & $20 \%$ & 2017 \\
\hline Twitter & $\begin{array}{c}\text { None } \\
\text { Specified }\end{array}$ & $\begin{array}{c}\text { None } \\
\text { Specified }\end{array}$ & $\begin{array}{c}\text { None } \\
\text { Specified }\end{array}$ & $\begin{array}{c}\text { None } \\
\text { Specified }\end{array}$ \\
\hline Vmware & $\begin{array}{c}\text { Not } \\
\text { Applicable }\end{array}$ & $\begin{array}{c}\text { Not } \\
\text { Applicable }\end{array}$ & $40 \%$ & 2050 \\
\hline Yahoo! & $\begin{array}{c}\text { None } \\
\text { Specified }\end{array}$ & $\begin{array}{c}\text { None } \\
\text { Specified }\end{array}$ & $\begin{array}{c}\text { None } \\
\text { Specified }\end{array}$ & $\begin{array}{c}\text { None } \\
\text { Specified }\end{array}$ \\
\hline \multicolumn{5}{|c|}{$\begin{array}{l}\text { a Cisco is seeking to source } 25 \% \text { of its electricity use annually from } \\
\text { renewable energy from } 2013-2017 \\
\text { b Information is specific to IBM's Austin, TX; Costa Mesa, CA; and Foster } \\
\text { City, CA facility. }\end{array}$} \\
\hline
\end{tabular}

\section{ENERGY PROFILES}

Profiling the energy consumption for network devices motivates manufacturers to implement green technologies and to achieve meaningful power savings. The energy profile is defined as the dependence of the energy consumption (in Watt-hours, Wh) as a function of four main factors: the traffic load through the device, the number of active ports, the line speeds, and firmware version [30].

Figure 5 shows various energy profiles depending on the traffic through the network devices [48]. Moreover, the casestudy calculation results show that by applying energy profile aware routing, a significant amount of energy and operational cost can be saved.

--On-Off: this energy profile corresponds to the characteristics of many existing devices. It is the most simple energy profile that fully empowers the network equipment when this is later turned on (e.g. the operation of traditional Ethernet switches). The energy consumption of this profile does not depend on the usage (i.e. actual traffic load).

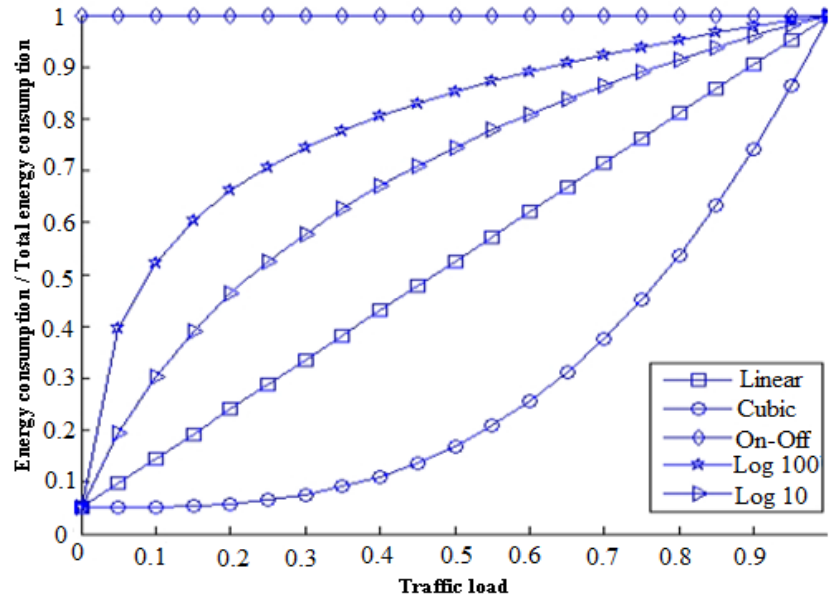

Figure 5. Proposed Energy Profile [48]

--Linear:devices belonging to this energy profile exhibit a power consumption that is proportional to their usage. Switch manufacturers such as Batcher, Fully_connected and Crossbar follow this energy profile [54]. Ideally, devices should have a linear energy profile. In addition, Barroso [55] introduced the propotional computing concept that may be applied to individual devices and components such as PCI slots and CPU cores to be into a sleep state when they are idle.

$--\log 10$ : this energy profile is an approximation of the profile produced by devices using the hibernation technique, so-called low-power idle proposal adopted in IEEE 802.3az Task Force [56]. Moreover, in [57], it was shown that the energy consumption can be as low as $10 \%$ compared to that of the On-Off energy profile.

$--\log 100$ : this energy profile corresponds to an intermediate function between $\log 10$ and On-Off representation. It might be a realistic end result when the proposed techniques by IEEE $802.3 \mathrm{az}$ are implemented.

--Cubic: this profile corresponds to equipment that adopts power reduction techniques like dynamic frequency scaling DVS (Dynamic Voltage Scaling) and DFS (Dynamic Frequency Scaling). In [58], Ethernet interface cards implementing DVS and DFS were demonstrated to reduce power consumption cubically.

Another study of energy consumpion profiles was introduced in [60]. Figure 6 depicts the different loaddependent energy profiles as well the optimised footprint that a device could present as a function of its utilisation. 


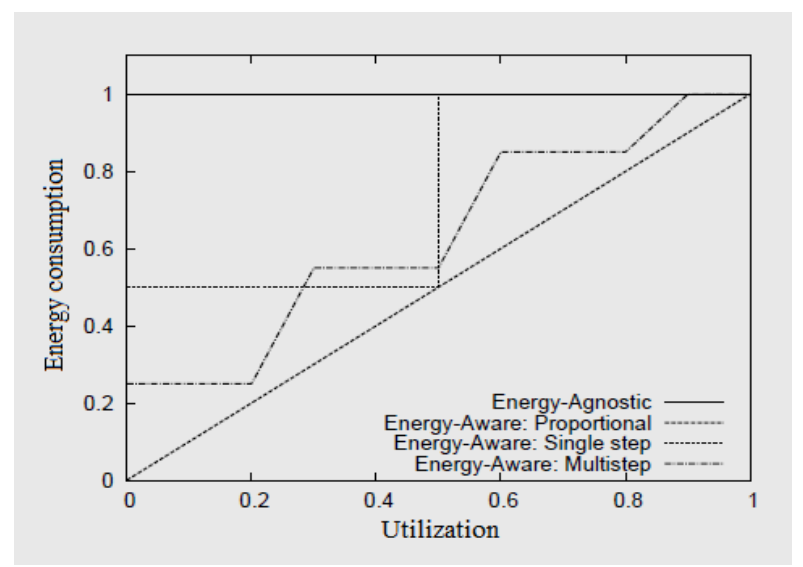

(a) Energy consumption as a function of utilisation

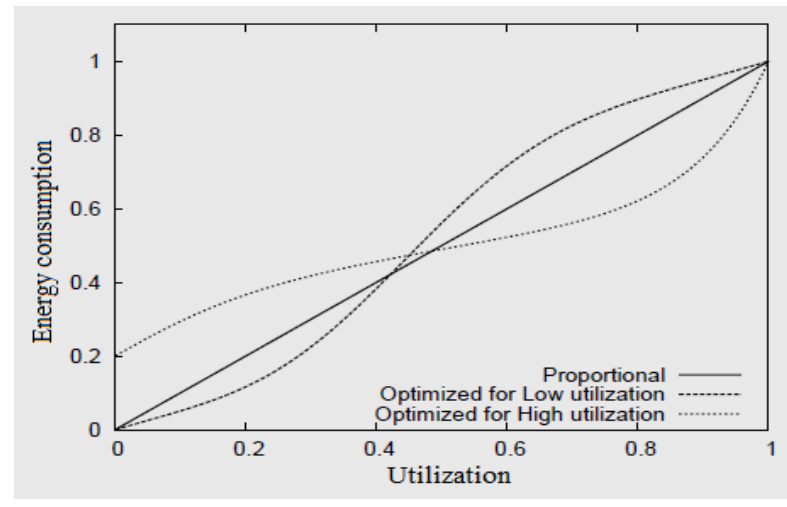

(b) Optimised footprint as a function of utilisation

Figure 6. Load-dependent energy consumption [55]

Another important energy profile model was introduced in [5]. This model is achieved through analytical framework. It allows estimating the energy profile of network devices, within diverse states, adopting two mechanisms, i.e. Adaptive Rate (AR) and Low Power Idle, described in Section V. Figure 7 shows an energy profile corresponds to a generic device that has four available AR states $(\mathrm{S})$. The power consumption of a device is assumed to have $S$ power states, which are thought to be ordered from the lowest energy consumption state $(s=0)$ to the most energy-hungry state $(s=S-1)$. Where $\gamma$ is the dependency parameter of idle optimisation from power states (if $\gamma=0$, idle logic is completely unaware of power states, if $\gamma=1$ there is no idle $\operatorname{logic}) . \lambda$ is the normalized value of traffic load incoming to the device. $\varnothing$ indicates the energy consumption of the device. $\sigma$ is a shape parameter of idle optimisation efficiency (ideal case, real case). $\xi$ represents how much is conservative the optimisation in terms of choosing the most suitable power state maintaining the desired QoS level for the incoming traffic. Finally, $v$ is a shape parameter of power states' energy consumption. The optimal profile simply consists of a piece-wise curve, composed by the most power saving parts of AR curves guaranteeing that the sum between the average incoming traffic load and a guaranteed threshold $(\xi)$ is lower than the maximum value of the service rate in AR states.

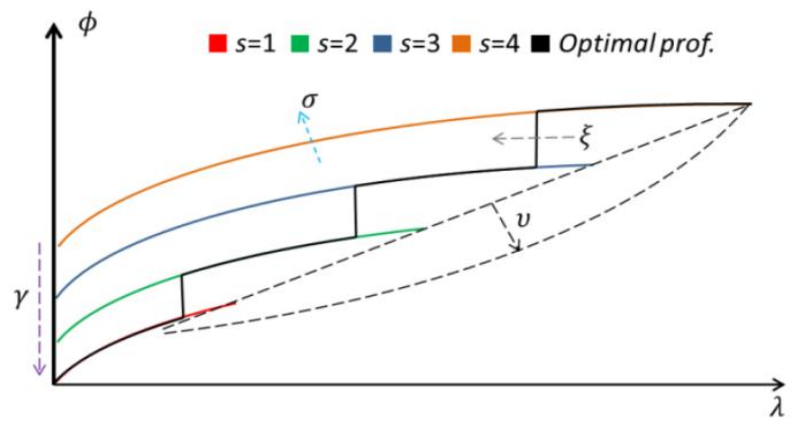

Figure 7. Energy profiles in various AR states in the presence of LPI primitives and the resulting optimal device energy profile[5]

Network design and traffic-engineering decisions can exploit the full energy-saving potential of network devices using information of load-dependent energy consumption and energy profiles. In this respect, a recent standardised interface referred as Green Abstraction Layer (GAL), has been approved by ETSI (the European Telecommunication Standards Institute) [124]. GAL interface enables energy management protocols to consistently determine which power management capabilities are available at the data plane, their potential effects on both energy consumption and network performance, and how to interact with them. [125] illustrates how GAL represents a multilayered (fourlayers) abstract model of the energy-saving capabilities for devices deploying local and network-wide control policies in a heterogeneous setting. Besides GAL, an energymanagement working group (EMAN) has been established by the IETF [126]. EMAN reuses existing works and investigates existing standards such as those from IEC (International Electronical Commission), DMTF (the Distributed Management Task Force), ANSI and others. The EMAN framework allows monitoring heterogeneous devices connected to a network to report their energy use over the time. For instance, the EMAN framework [127] describes how energy information (measurement, characteristics, identification) can be retrieved from IPenabled devices using Simple Network Management Protocol (SNMP) with the help of MIB (Management Information Base) modules.

\section{COST AND POWER CONSUMPTION IN OPTICAL NETWORK}

Carrier-grade networks are provisioned in order to meet a set of requirements, as mentioned before, resiliency, scalability, quality of service and service management of the network. All these critical aspects are commonly supported through optical networks. Besides, deploying optical switching technologies is considered as a promising solution to enable energy efficiency in the core/transport segment of the internet. These reasons highlight the necessity to identify the cost and the power consumption of optical networks. 
Hence, this section defines different architectures suitable for carrier-grade networks, especially those employing optical WDM (Wavelength Division Multiplexing) technologies. Then, we present their corresponding cost (CAPEX) and energy consumption (OPEX) models. WDM is a technology that is most probably deployed in broadband area networks. It multiplexes multiple optical carrier signals on a single optical fibre by using different wavelengths of light. WDM technology utilises Optical Cross-Connect (OXC) devices to perform switching and accelerates routing at the optical layer.

\section{A. Network Node Architectures}

The core/metro optical network architecture can be opaque and transparent $[102,103]$. In the opaque architecture (Figure 8), all optical signals carrying traffic undergo an optical to electronic to optical (OEO) conversion (and viceversa) at every node in the network. On the other hand, in transparent and translucent architecture (Figure 9), OEO conversion can be avoided by simply allowing in-transit traffic to bypass optically intermediate nodes. The OEO conversion represents the largest operational cost for optical fibre networks.

Different opaque architectures are used in practice among others: SONET/SDH, basic IP over WDM (BIPoWDM), and carrier-grade Ethernet technologies. SONET/SDH is the legacy architecture in MAN. It is a circuit-based technology that can aggregate low-bit rate traffic streams from metro networks into high bandwidth pipes of core networks [104]. In SONET/SDH nodes, all switching in the data plane takes place in the electronic domain. In the next generation of optical transport networks, SONET/SDH will progressively disappear because it is no longer efficient to sustain today's data traffic progress. In BIPoWDM) architecture, inside each node an OXC is interconnected to the IP router. This architecture is also known as lightpath non-bypass design. The routers are interconnected by point-to-point optical fiber links; traffic flows undergo $\mathrm{O} / \mathrm{E} / \mathrm{O}$ conversion at every intermediate node. Moreover, in order to provide carrier-grade service, extensions to regular Ethernet switching have led the definition of what is known as carrier grade Ethernet, taking advantages of its simplicity, scalability and effective cost. Ethernet-over-fibre supports a link rate of up to $100 \mathrm{Gbit} / \mathrm{s}$. Carrier Ethernet switch performs OEO conversion of every wavelength as in a SONE/SDH node. It can use DWDM (Dense WDM) technology. DWDM is used to transmit many optical channels on the same fibre component.

Transparent architectures are expected to enable significant power conservation, due to the minimum needed number of OEO conversions. Among the transparent architectures one can consider: transparent Tp-IPoWDM (Tp-IPoWDM) and hub-based. This architecture is also known as lightpath bypass design. In Tp-IPoWDM, each node is equipped with micro-electrical mechanical systems (MEMS) and as for the opaque case the IP router is interconnected to an $\mathrm{OXC}$ device. If signal regeneration is required, the lightpath has to be dropped and the matching traffic sent to the IP router to be processed. When no regeneration is required, a significant amount of energy can be saved because the traffic can be switched directly in the optical domain by OXCs (bypassed), conserving the capacity of the wavelength [103].

In the hub-based architecture, the traffic sent/received by an access node is either local (i.e. transmitted from/to one of the access nodes in the same metro optical network) or intermediate (i.e. directed to/from the Internet). As most of the traffic is transit traffic, the transparency is carried out by permitting every access node to have a direct light-path (i.e. specific wavelength channel) to the hub node. Evidently, the hub-based architecture is suitable for today's network traffic states, i.e., the traffic is either terminated or originating from the Internet. Although the hub-based architecture avoids OEO conversion costs at intermediate nodes, it is insufficiently powerful especially in the case of dynamic traffic scenarios, since the qualities of optical signals degrade as they travel through numerous optical components. To overcome these impairments, sharing bandwidth of each channel between multiple source-destination nodes should be implanted.

Interested approaches are suggested in order to optimise the energy consumption in transparent networks $[175,176,180]$.

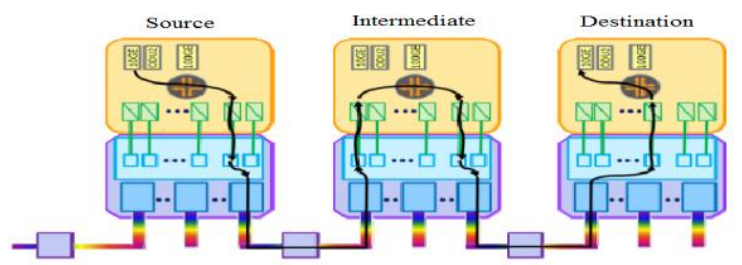

Figure 8. Opaque network architecture [106]

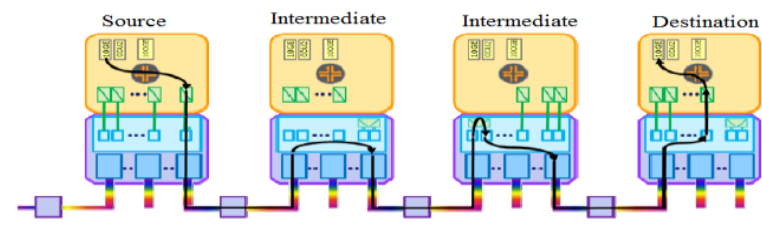

Figure 9. Transparent network architecture [106]

\section{B. Analytical model for the energy consumption of a WDM optical network}

The estimation of the energy consumption of the physical infrastructure resources is highly dependent on the network architecture employed and the technology made.

In this section, we present an analytical model that considers the WDM optical network architecture employing wavelength selective switches using MEMS [107].

The overall energy consumption model is based on the active elements of the network that can be classified as OXC nodes and transmission link-related elements. Each OXC node comprises a set of active and passive elements. Figure 10 illustrates the assumed $\mathrm{OXC}$ architecture. The passive elements incorporated in these nodes are: the multiplexers (MUX) and demultiplexers (DEMUX), while the active elements are: the photonic switching matrix, one ErbiumDoped Fibre Amplifier (EDFA) per output fibre port, one Optical-Electrical-Optical (OEO) transponder per output 
wavelength port and one transmitter $(\mathrm{Tx})$ - receiver $(\mathrm{Rx})$ per

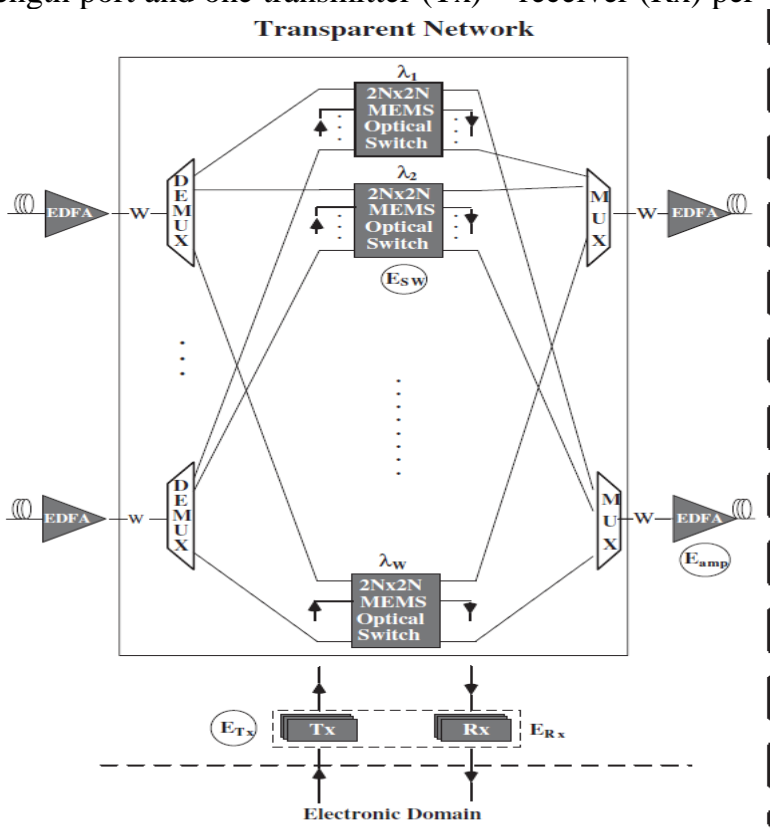

(a) lightpath located at the add and drop ports of the OXC. Opaque Network

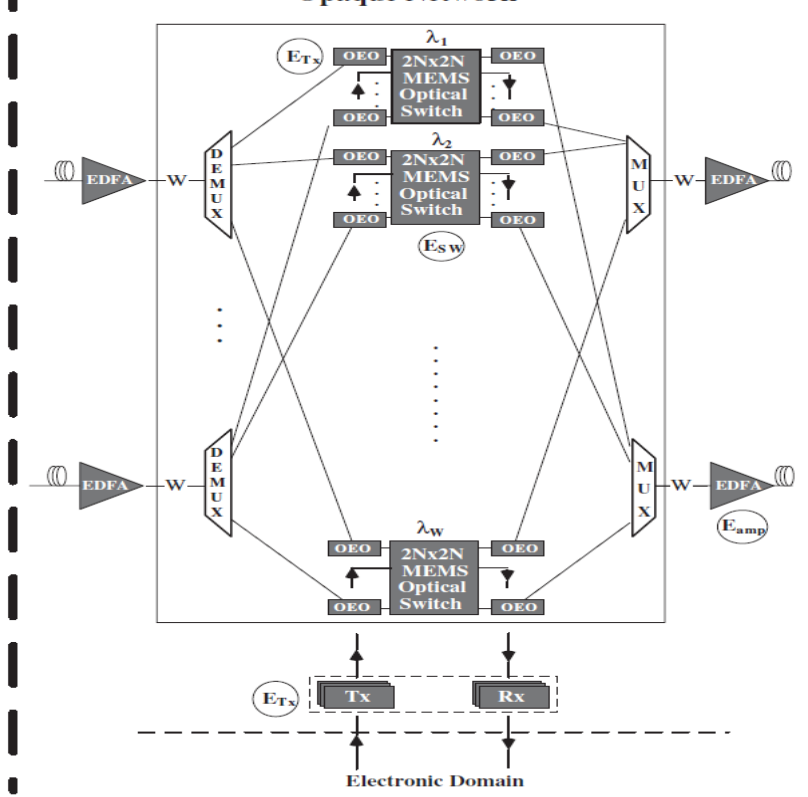

(b)

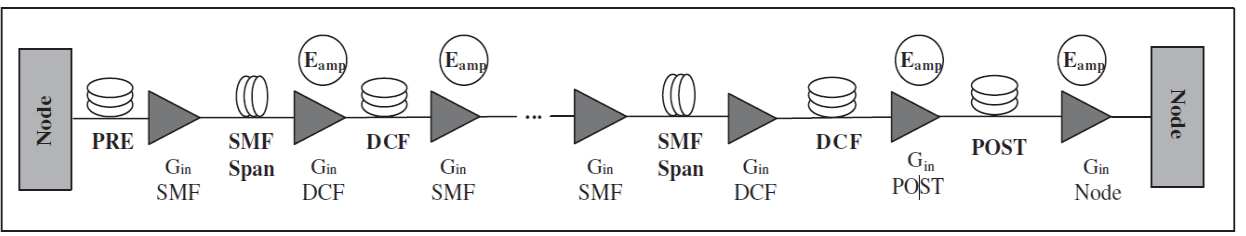

(c)

Figure 10. OXC architecture [107]

As the assumed OXCs are symmetrical, the number of bypass ports of the OXC is calculated as the product of the number of input fibres and the maximum number of wavelengths that a fibre can support.

The overall network power consumption is determined by the power consumption of the individual OXCs and fibre links (which includes the installed optical amplifiers). The power consumption of node $n\left(P_{O X C_{n}}\right)$ depends on the four active elements in the OXC: the power of the switching fabric $\mathrm{P}_{\mathrm{sf}}$, power of OEO transponders for transmission $\mathrm{P}_{\text {Tran, }}$, the power of the wavelength converters $\mathrm{P}_{\mathrm{Con}}$, and the power of the optical amplifier $\mathrm{P}_{\text {Amp. Equations }}$ (1) - (4) describe the dependence of the power consumption of the node on the individual elements power consumption.

$$
\begin{aligned}
& P_{S F}=\text { ports }_{\text {total }} \times P_{\text {port }_{\text {pair }}} \\
& \quad=\left(\text { ports }_{\text {th }}+\text { ports }_{a / d}\right) \times P_{\text {port_pair }_{\text {_p }}} \\
& P_{\text {Tran }}=\text { ports }_{a / d} \times P_{T X / R x} \\
& P_{\text {Con }}=\text { ports }_{\text {th }} \times P_{\text {transpoder }} \\
& P_{\text {Amp }}=\left(f_{\text {in }}+f_{\text {out }}\right) \times P_{E d f a}
\end{aligned}
$$

The power of a switch fabric is computed as the number of ports (i.e. the sum of bypass ports ports th $_{h}$ and add/drop ports ports $_{a / d}$ ) multiplied by the power consumed by each switch port.

The power related to the OEO transponders for transmission is the product of the add/drop ports and the power related to the transmission device $P_{T x / R x}$. The power consumption of the installed amplifiers is computed as the product of the number of incoming and outgoing fibres $\left(f_{\text {in }}\right.$ and $\left.f_{\text {out }}\right)$ and the power related to the optical amplifiers.

Figure 15c illustrates the fibre link model [107], where the only power-consuming elements are the optical amplifiers installed per span. The maximum span length (span) is expected to be $80 \mathrm{~km}$. Thus the power consumption $P_{l}$ of a fibre link $l$ is length-dependent and is calculated as follows:

$$
P_{l}=\left\lfloor\frac{\text { length }(l)}{\operatorname{span}}\right\rfloor P_{E d f a}
$$

Finally, the total energy consumption of the physical infrastructure of N OXC nodes is calculated as follows:

$P_{N e t}=\sum_{n \in N} P_{O X C_{n}}+\sum_{l \in L} P_{l}$ 


\section{Cost and power consumption}

The CAPEX and OPEX of optical WDM architectures has been the subject of several case studies [102,106-108]. These analyses are often based on linear programming models and heuristic approaches.

[102] provide models for evaluating the cost, power and traffic capacity for opaque and transparent architectures. The total power consumption and the cost of an opaque and a transparent network node are determined by subdividing the node into its main functional blocks: the base node and the equipment related to the physical layer interfaces. The base node consists of the chassis, the mechanical assembly, the switching matrix, the cooling functionalities, the power supply, the control and the management functions. Every base node has a limited number of slots for physical layer interfaces.

Figures 11 and 12 recapitulate the normalised cost and power consumption for the components of Ethernet carriergrade switches and OADM (Optical add-drop Multiplexer), respectively.

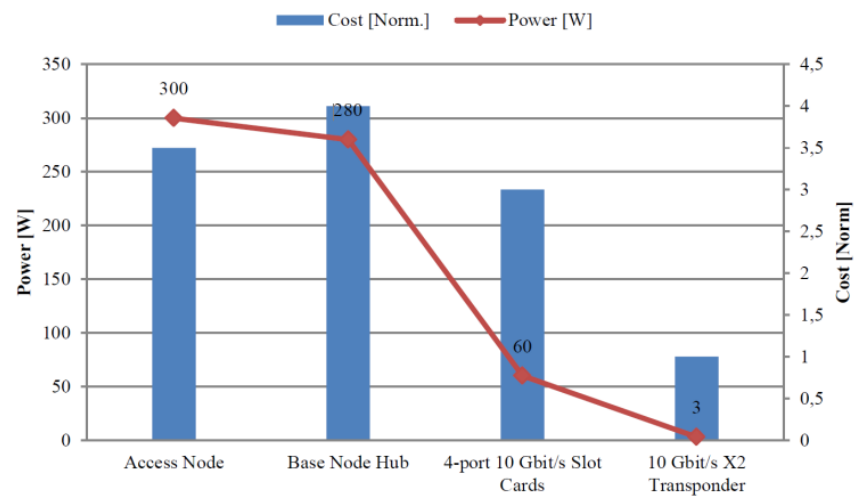

Figure 11. Normalised cost and power consumption for Ethernet Carriergrade nodes

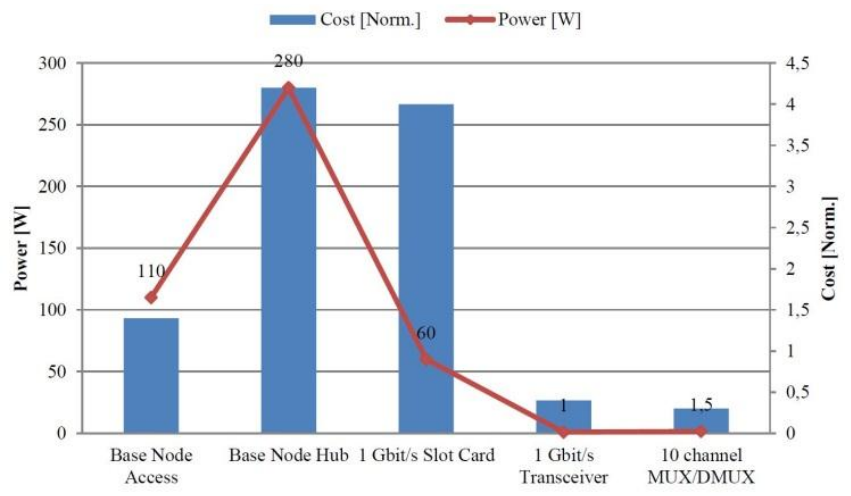

Figure 12. Normalised cost and power consumption for an OADM

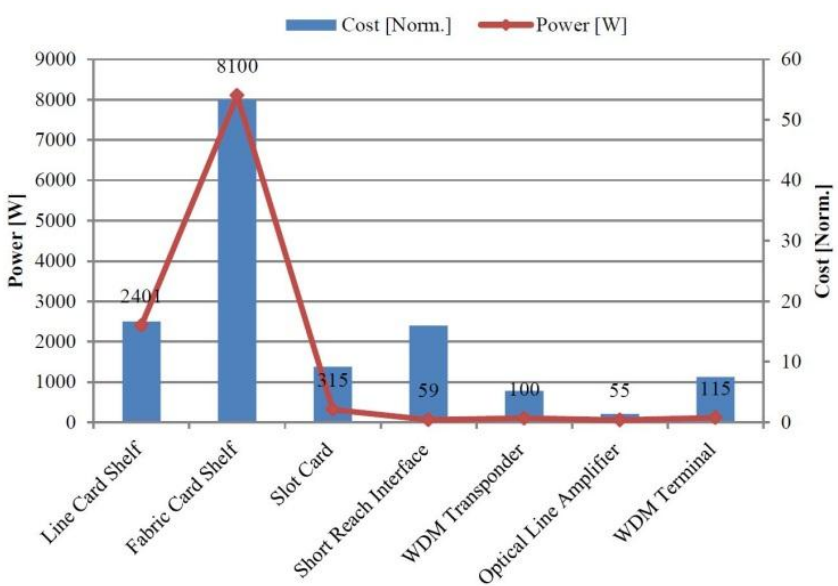

Figure 13. Normalised cost and power consumption of WDM network components

[107] models the cost of the power consumption of WDM network components in terms of cost and operational power by signal transmission, as reported in Figure 13.

The estimation of the power consumption of the network components is highly dependent on the network node architecture and the network topology. However, packet transmission time or the link load can be crucial parameters to compute the energy consumption in networks that implement power-saving mode, as demonstrated in [109]. These authors provide an analytical model to estimate the power consumption of optical Ethernet links that implement a power-saving mode. The model splits the time into discrete time intervals and assumes that the transition times between modes are a multiple of the frame transmission time.

Measuring the overall power consumption of carriergrade networks before and after applying green approaches is an efficient metric to evaluate the applied techniques. For instance, in data centres, the most frequently used metric is power usage effectiveness (PUE). PUE is the ratio of the total energy used by a data centre, including IT equipment, to the energy consumed by the IT equipment only.

Along with PUE, three metrics allow the measurement of the energy efficiency of carrier grade networks: IT Equipment Utilisation (ITEU), IT Equipment Energy Efficiency (ITEE) and Green Energy Coefficient (GEC) [115].

$I T E U=\frac{\sum \text { Measured energy consumption of IT equipment }}{\sum \text { Rated energy consumption of IT equipment }}$

$I T E E=\frac{\sum I T \text { equipment rated } \text { work capacity }}{\sum \text { Rated energy consumption of IT equipment }}$

$G E C=\frac{\sum \text { Energy input from renewable resources }}{\sum \text { Energy consumption of operating network }}$

In this section, we have described the main node architectures in carrier-grade networks and provided illustrative examples of how to measure overall network 
power consumption, as well as the energy efficiency. Going further, more emphasis is given regarding saving energy in optical networks; among others: [175-190].

\section{TAXONOMY OF GREEN APPROACHES}

This section depicts the relevant classifications provided in the literature. We give a comparison and insights of the presented classifications. Then, we provide our taxonomy for current green networking approaches.

Before presenting our taxonomy and discussing the different approaches for saving energy in networking, we present the most pertinent surveys in the literature.

\section{A. Existing classification of green networking approaches}

In the research literature, there are extensive surveys from slightly different points of view that cover approaches related to green networking [4,27,37, 128, 129].

The survey in [42] provides a comprehensive review of the techniques and solutions that aim to improve the energy efficiency of large-scale distributed systems. The authors classify existing research into four categories: (i) hardware, (ii) shutdown, (iii) slowdown, and (iv) coordination and network-wide solutions. Hardware studies approach energy efficiency by exploiting optimal equipment architecture or by designing novel energy-efficient technologies. Shutdown approaches are devoted to putting idle components to sleep. Slowdown approaches are devoted to scaling the transmission speed of interfaces dynamically according to the needs. Coordination refers to the management of network power through the improvement in protocols and architecture design.

Another survey gives a review and taxonomy of relevant techniques applied in wired networking [27]. Their taxonomy identified four branches of green networking research: (i) adaptive link rate, (ii) interface proxying, (iii) energy-aware infrastructure, and (iv) energy-aware applications. Adaptive link rate refers to scaling down the link rate proportional to the traffic load, even to zero i.e. to the sleep state. Interface proxying refers to reducing power consumption at the application layer while the network connectivity is maintained due to the proxy structure. The energy-aware infrastructure category includes methods that adopt energy consciousness throughout the network design stage. The final category, energy-aware applications, focuses on studies that call for energy consciousness in software design.

A detailed survey on emerging technologies, standards efforts, and projects is given in [4]. These authors identified three categories of green networking research: (i) reengineering, (ii) dynamic adaptation, and (iii) sleeping/standby. The first category intends for network devices and architecture to be optimised or to design new network equipment completely. The second category, dynamic adaptation, suggests approaches that work on modulating the capacities of network device resources in order to meet the actual services and traffic requirements Finally, sleeping/standby is founded on power management basics that allow network equipment (or parts of them) to be switched off, to enter very low power states.

A recent survey [128] mainly focuses on energy efficient solutions for cloud-based networking components, making use of the system literature review (SLR) research method [129]. Four groups of methodologies have been identified based on the networking component granularity: (i) Data Center (DC) layer, (ii) Application (App) layer, (iii) Network layer, and (iv) Device layer.

Additionally, the green traffic engineering approaches have been studied from the perspective of optimisation modelling issues in the survey article [130]. The approaches have been split into two main groups: (i) flow-based routing and (ii) shortest path routing. We draw Table VI to compare this work with other related works.

TABLE VI

FOCUS CONCEPTS OF THIS STUDY VIS-A-VIS RELATED SURVEYS

\begin{tabular}{|c|c|c|c|c|c|c|c|}
\hline \multirow{2}{*}{$\begin{array}{l}\text { Survey } \\
\text { paper }\end{array}$} & \multirow[b]{2}{*}{ Key aspect } & \multicolumn{2}{|c|}{ Node level } & \multicolumn{4}{|c|}{ Network level } \\
\hline & & $\begin{array}{l}\text { Hardware } \\
\text { optimisation }\end{array}$ & $\begin{array}{l}\text { Software } \\
\text { optimisation }\end{array}$ & Proxying & Virtualisation & $\begin{array}{l}\text { Traffic engineering } \\
\text { with traditional } \\
\text { protocols }\end{array}$ & $\begin{array}{l}\text { Traffic engineering } \\
\text { with SDN }\end{array}$ \\
\hline [4] & $\begin{array}{l}\text { Engineering of wired and } \\
\text { wireless network devices }\end{array}$ & $\checkmark$ & $\checkmark$ & $\checkmark$ & $\checkmark$ & - & - \\
\hline [37] & $\begin{array}{l}\text { Techniques that improve } \\
\text { energy efficiency of computing } \\
\text { and wired network ressources }\end{array}$ & $\checkmark$ & $\checkmark$ & $\checkmark$ & $\checkmark$ & - & - \\
\hline [27] & $\begin{array}{l}\text { Green emergent technologies in } \\
\text { wired and wireless networks }\end{array}$ & $\checkmark$ & $\checkmark$ & $\checkmark$ & $\checkmark$ & - & - \\
\hline [128] & $\begin{array}{l}\text { Energy efficient solution in } \\
\text { cloud-based networking }\end{array}$ & $\checkmark$ & - & - & $\checkmark$ & $\checkmark$ & $\checkmark$ \\
\hline
\end{tabular}




\begin{tabular}{|c|c|c|c|c|c|c|c|}
\hline$[130]$ & $\begin{array}{c}\text { Optimisation modelling for } \\
\text { energy-aware traffic } \\
\text { enginering in wired and } \\
\text { wireless networks }\end{array}$ & - & - & - & - & $\checkmark$ & $\checkmark$ \\
\hline $\begin{array}{c}\text { This } \\
\text { study }\end{array}$ & $\begin{array}{c}\text { Energy consumption } \\
\text { measurement and techniques } \\
\text { that enhance energy efficiency } \\
\text { for wired (main focus and } \\
\text { carrier grade networks }\end{array}$ & $\checkmark$ & $\checkmark$ & $\checkmark$ & $\checkmark$ & $\checkmark$ & $\checkmark$ \\
\hline
\end{tabular}

\section{B. Classification of green networking approaches}

Merging the main points of the three reviews above, we propose a new taxonomy of energy-aware strategies for green networks, as illustrated by Figure 14. Our classification is focused on two main categories: node level and network level. In the former category, two kinds of optimisation-based strategies are proposed: either energy optimisation strategies, which are applied on hardware design; or energy optimisation strategies, which are applied on software functions. In the latter category, the energysaving problem is treated at network level; this category can be divided into three main sub-categories: proxying, virtualisation, and traffic engineering. The final sub-category can be further classified into two kinds of traffic engineering: soft-defined networks and traditional protocols.

\section{1) Node level}

\section{a) Hardware optimisation}

A large number of studies and projects have introduced energy-aware technologies to exploit them efficiently inside network equipment design [59-64]. The main focus of these projects is to reduce the power consumption of specific components per node such as CPUs [24], disks [23] and NICs [22].

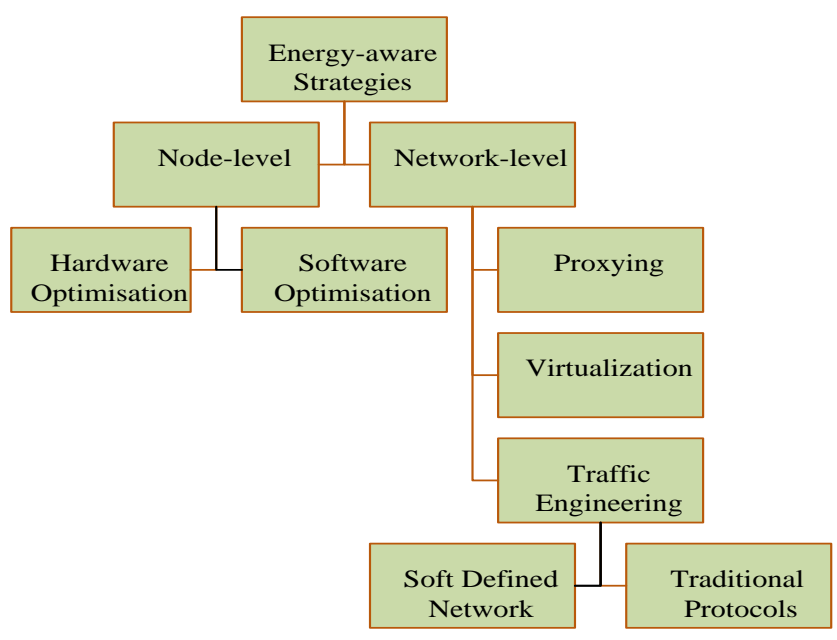

Figure 14. Taxonomy of green networking approach
The basic technique of these approaches is to scale the voltage and frequency (i.e. power consumption) proportional to the service demand. For example, link transmission rates between components can be modulated to limit the energy consumption and to meet the actual traffic requirements. This hardware management capability is usually referred to as power scaling, AR (Adaptive Rate) or as ALR (Adaptive Link Rate).

The ALR technique was first described by Gunaratne et al in 2005 [131] target to adjust NICs speed to the effective workload. Two main methods keys are needed to develop the ALR technique. The first is to determine exactly how the link data rate is switched, namely ALR mechanism. The second defines an ALR policy to decide when to switch the link data rate, in such a way as to minimise the increase of the packet delay and to maximise the power saving. Thus, the performance tradeoff in ALR techniques is packet delay versus power saving.

In order to avoid the time of sending a long frame preamble, Gunaratne et al in 2008 [65] proposes a faster handshake and resynchronisation mechanism, which is implemented using Ethernet MAC frames, able to execute them effectively in less than $100 \mu$ s at $1 \mathrm{Gbit} / \mathrm{s}$. Initially, the link that determines the need to increase or decrease its data rate requests a data rate change using ALR Request MAC frame. Then, the receiving link replies to the data rate change request with either an "ALR ACK reply" if it agrees to change the data rate, or an "ALR NACK reply" if it does not agree. After the ALR ACK response, the link data rate can be switched and the link resynchronised. The total time of the handshake and resynchronisation process can be less than $100 \mu$ s for $1 \mathrm{Gbit} / \mathrm{s}$ Ethernet.

In [66], a Markov model is developed where the Ethernet link data rate is a function of link use. This proposal identifies high-buffer and low-buffer thresholds. The use of two thresholds avoids frequent oscillations between two rates. When the buffer occupancy reaches the high-buffer threshold, the link rate is increased to a higher value, and when it goes under the low-buffer threshold, the link rate is decreased. The difficulty lies in finding good values for these thresholds in order to avoid packet losses and oscillations, since switching between rates takes time.

A similar mechanism, called the Dynamic Adjustment of Link Width (DAWL), suggested in [67], also uses dualthreshold based on link utilisation. These authors advocate that the distribution of operating rates and their 
corresponding power consumption significantly influences the efficiency of the adaptation techniques.

In addition, components can be exchanged by more energyefficient mechanisms such as the replacement of electrical components with their corresponding item in the optical domain [68-70].

In addition to ALR technique, LPI is also an energy saving technique delivered by the hardware level. Indeed, the IEEE 802.3az task force [56] defines the LPI technique to offer the standardisation solution, Energy Efficient Ethernet (EEE), to improve Ethernet NICs and switches. EEE defines two operational modes for transmitters and receivers: active mode and LPI mode (i.e. idle link period). Compared to the active mode, LPI mode brings the energy consumption down to $10 \%$. The basic concept is to transmit data as fast as possible; to let then the device spends a significant fraction of time in low power or (sleep) mode. However, the transition between the different modes creates an overhead to every burst of one or more packets sent consecutively. As a result the energy saving achieved is strongly dependent on the traffic pattern and packet size distribution. In other words, the average power consumption of Ethernet interface is heavily dependent on the fraction of time the interface spends in LPI, active, and transitioning between states.

The EEE performance is improved in particularly using a technique called burst transmission [132] or packet coalescing [57], which allow overcoming the effect of EEE overhead. The idea of packet coalescing technique is to aggregate packets in a buffer until either the buffer is full or the timeout expires. However, the buffer size and the coalescing timeout setting strongly affect the trade-off in EEE performance. Since then, several works addressed modeling and performance analysis of EEE based on various traffic parameters and with and without consideration to packet coalescing [133-139]. In [133] authors provide an evaluation of static and dynamic coalescing for EEE in which buffer size and timeout are fixed (static) and adapted to traffic pattern (dynamic). The results show that static coalescing and dynamic coalescing algorithms achieve similar power saving and delay tradeoffs. Therefore, static coalescers are preferable for real implementation due to their low complexity.

The work in [135] provides an accurate traffic model with GI/G/1 queues for both frame and burst transmissions. The model allows predicting the average energy saving as well as the impact of sleeping algorithms on packets delay. However, this model is valid only for unidirectional traffic and specifically designed for the case of $10 \mathrm{Gbit} / \mathrm{s}$ links. Also, the work in [139] provides a model that can be used for three available EEE links: 100BASE-TX, 1000BASE-T and 10GBASE-T. By analysing energy consumption and various network performance indexes in closed form, i.e., without upper bound and lower bound approximations, makes the model suitable to be adopted in optimisation frameworks. This model can be used and useful for dimensioning interfaces during the design phase of a datacenter.
Figure 15 shows the transitions between modes as defined in EEE, as well as, it indicates a qualitative indication of the energy consumption $E(t)$ for the different periods. However, the sleep and wake-up periods are intensely greater for small frames and higher speed [139].

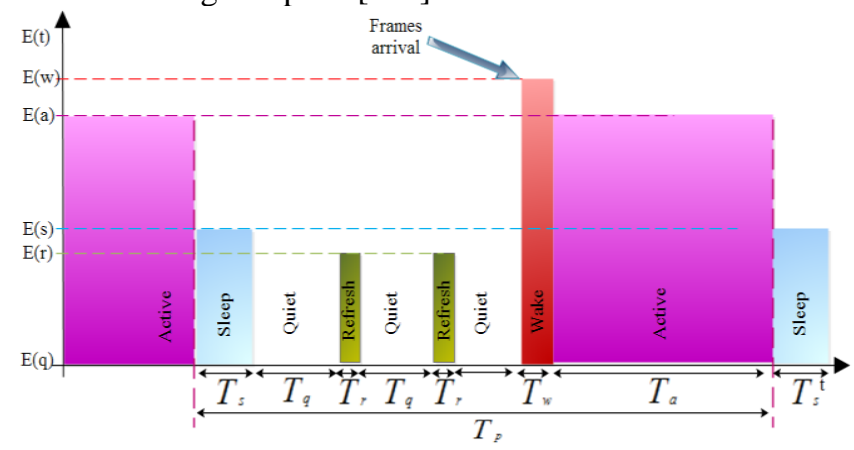

Figure 15. Relative power consumption for different periods of EEE

$T_{S}$ is the sleep time required to enter LPI mode. Once the device enters LPI mode, it stays quiet during large period $T_{q}$, and only sends signals during short period $T_{r}$ (refresh time). $T_{r}$ permits faster wake-up time and maintains alignment between Transmitter (TX) and Receiver (RX). Once frames arrive, the link should exit the LPI mode taking $T_{w}$ seconds to be in active mode during $T_{a}$ seconds. Finally, refers to the average duration idle/busy cycle.

In [71], the energy consumption of representative optical and wireless devices that are provided from manufacturers' data sheets for optical access networks is analysed. The energy consumption model is defined as a function of the access rate to users, and is strongly based on traffic estimation. This analysis takes into account various network technologies (DSL, HFC, PON, FTTN, point-to-point optical system, UMTS (WCDMA), and WiMAX). They demonstrate that passive optical access networks (PONs) and point-to-point optical networks are the most energy-efficient of the available access technologies.

\section{b) Software optimisation}

Current technologies, including software, consider energy efficiency in their operating systems and software applications. The running software hides various processor techniques that might waste electricity. Research demonstrates that operating systems having a heterogeneous power consumption could be improved to consume less power [72,73]. In other words, device consumption within a different version of the same operating system can have considerable variation.

Software power management is promoted by the Advanced Configuration \& Power Interface (ACPI) standard [74]. The main idea consists of the operating system managing power supply for each component, in order to avoid unnecessary power consumption. This standard provides an interface between the hardware and software layers, by modelling the different set of working and idle states (C-states). C-states are described as follows: the $C O$ power state is an active power state where the CPU performs tasks, while the $C l$ to $C n$ power states are processor sleeping or idle states, where 
the processor consumes less power and dissipates less heat. Furthermore, as the sleeping power state $(\mathrm{Cl}, \ldots, \mathrm{Cn})$ becomes deeper, the transition between the active and the sleeping state (and vice versa) needs longer time. Table VI shows that the transition between the $C O$ and $C 1$ states requires only a few ns, while $50 \mu$ s are required for the $C 3$ state. Practically, the higher the index of $\mathrm{C}$ states is, the lower will be the power consumed, and the heat spent.

TABLE VII. INDICATIVE ENERGY SAVING AND TRANSITION TIMES FOR COTS ( COMMERCIAL OFF-THE-SHELF) PROCESSOR C-STATES [G2010]

\begin{tabular}{|c|c|c|}
\hline C-state & $\begin{array}{c}\text { Energy-saving with } \\
\text { respect to the C0 state }\end{array}$ & Transition times \\
\hline$C 0$ & $0 \%$ & - \\
\hline$C 1$ & $70 \%$ & $10 \mathrm{~ns}$ \\
\hline$C 2$ & $75 \%$ & $50 \mu \mathrm{s}$ \\
\hline$C 3$ & $80 \%$ & $160 \mu \mathrm{s}$ \\
\hline$C 4$ & $98 \%$ & $200 \mu \mathrm{s}$ \\
\hline$C 5$ & $99 \%$ & Unknown \\
\hline$C 6$ & $99.9 \%$ & \\
\hline
\end{tabular}

Similar research has attempted to hide the details of energy-saving techniques that are implemented in data-plane, by creating middleware running in computing resources [75]. In fact, middleware can influence the power consumed by the platforms as it maps physical resources and the user's tasks [76]. For that matter, Blanquicet et al. [77] provided the means for the management of applications to measure the power consumption of IT equipment and to indicate the state at which their components operate.

\section{2) Network level}

\section{a) Proxying}

The sleep proxy concept was introduced in 1998 with the pioneering work of Christensen [78]. A sleep proxy scheme enables idle end devices such as PCs to pass into sleep state dealing with their potential loss of network connectivity. Before going to sleep, the idle PC transfers its network presence to the proxy, and after that the proxy responds to non-urgent messages on behalf of the sleeping node/PC and wakes-up the node/PC only if required. It handles network requests such as ARP, ICMP and DHCP. The structure of the NPC (Network Proxy Connectivity) scheme is shown in Figure 16. Since the proxy consumes much less than the CPU of the PC and one proxy can be shared by many PCs, the energy consumption of the system is reduced.
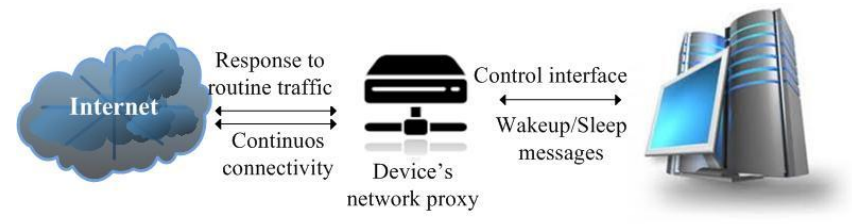

Figure 16. Network connectivity proxy

Another proxy structure can be implemented in NICs. This solution is referred to as interface proxying, and does not need external proxy devices. NIC proxying implements a small handling to the incoming traffic: each NIC handles non-urgent traffic, while a whole node will wake up when urgent traffic requiring further computation is received. In [79], a framework is proposed to implant NIC proxying over existing hardware. It supports a line speed of up to $1 \mathrm{Gbit} / \mathrm{s}$ in its software implementation (on the Smart-NIC), and up to $10 \mathrm{Gbit} / \mathrm{s}$ in its hardware implementation. The hardware implementation consumes only $25 \%$ of the power consumed by the software implementation. For higher energy savings, it should reduce the number of device wake-ups and define longer sleeping intervals.

[80] analysed the possibility of easily deploying SleepServer architecture, i.e. dedicated on-demand proxy servers. The proposed architecture allows end hosts to utilise power saving modes more frequently. SleepServers use a very simple application agent on the end hosts. They are easily deployable because they do not necessitate any changes to current hardware, software or networking structure. [81] implements four different types of proxies with increasing complexity. They demonstrate that simple approaches are not sufficient to achieve the potential energy saving. A significant implementation is vital to handle broadcast traffic accurately and take into account the residential setting. The ECMA-393 standard [141], namely ProxyZzzy, have been recently introduced to provide an overall architecture and describe a common way to implement proxy functionality. This standard specifies essentially: capabilities that a proxy may expose to a host, information that must be exchanged between a host and a proxy, and proxy behaviour for IEEE 802.3 and IEEE 802.11. However, it is neutral about communication mechanisms between host and proxies as well as with an external proxy. Also, it does not specify a common interface for monitoring the proxying operations. Finally, to estimate the potential savings, authors in [142] analysed real patterns for home and office environment of proxying network connections. They provide full spectrum of deployment solutions, as well as consider both on-board (like NICs) and external proxy (like switches and routers) implementations.

\section{b) Virtualisation}

The virtualisation of physical resources combines a set of mechanisms that enable the operation of more than one service within the same machine. Applying virtualisation to computing resources brings many benefits such as efficient hardware utilisation and resource provisioning on demand, besides the reduction of both CAPEX and OPEX costs. The virtualisation technique is considered as a mature research field [82]. In fact, it occurred very frequently in data centres. Because rack space and power are expensive, deploying additional servers and routing entities without an increase in rack space is very much needed. Multiple resources and applications within the same organisation (like servers, firewall, storage, and network entities) are on the same physical device and hence employ virtualisation to assure the proper functioning of each resource and application.

The routers are defined to be isolated logical router processes and act like a physical router. Actually, these routers do not carry full internet routes, and the flows have a 
tendency to inferior speed due to bandwidth sharing on the servers. In [83], the authors focus on two main techniques for creating virtualised routing entities as defined by their physical and operational characteristics. A HardwareIsolated Virtual Router (HVR) has hardware-based resource isolation between routing entities, whereas a SoftwareIsolated Virtual Router (SVR) comprises software-based resource isolation between routing entities. Table VII presents a comparison of the two virtualised routing techniques.

In [84] VirtualPower is presented, a power management capability for virtualised systems that combined software and hardware scaling methods to control the power consumption of a given platform. The proposed infrastructure of the VirtualPower advocates two basic ideas. The first is to present VM guests with what appears to be a rich set of software states available to their applicationspecific policies, termed VPM states. The second is to use the state changes requested by VMs as inputs to virtualisation-level management policies.

\begin{tabular}{|c|c|c|} 
TABLE VIII. & $\begin{array}{c}\text { COMPARISON OF VIRTUALISED ROUTING } \\
\text { TECHNIQUES[83] }\end{array}$ \\
\hline Category & $\begin{array}{c}\text { Hardware-Isolated } \\
\text { Virtual Router }\end{array}$ & $\begin{array}{c}\text { Software-Isolated } \\
\text { Virtual Router }\end{array}$ \\
\hline $\begin{array}{c}\text { Control plane resources } \\
\text { (CPU, memory) }\end{array}$ & Dedicated & Shared \\
\hline $\begin{array}{c}\text { Data plane resources ( } \\
\text { forwarding engines, } \\
\text { queues) }\end{array}$ & Dedicated & Shared \\
\hline $\begin{array}{c}\text { Chassis resources (power } \\
\text { supplies, blowers, fabric) }\end{array}$ & Shared \\
\hline $\begin{array}{c}\text { Management } \\
\text { configuration }\end{array}$ & Dedicated & $\begin{array}{c}\text { Typically shared, but } \\
\text { varies depending on } \\
\text { degree of virtualisation }\end{array}$ \\
\hline $\begin{array}{c}\text { Connection between } \\
\text { virtualised routing } \\
\text { entities }\end{array}$ & $\begin{array}{c}\text { Typically external } \\
\text { possibly external }\end{array}$ \\
\hline $\begin{array}{c}\text { Per-chassis scalability } \\
\text { (routing adjacencies, } \\
\text { prefixes) }\end{array}$ & $\begin{array}{c}\text { Increased with additional } \\
\text { logical routers }\end{array}$ & $\begin{array}{c}\text { Unaffected by additional } \\
\text { virtual routers }\end{array}$ \\
\hline
\end{tabular}

They also evaluate the dynamic power consumption by a dual-core chip at different levels of frequency and voltage, and suggest that such a solution be coupled with a software scheme. On the other hand, [85] reported that the sole use of virtualisation does not guarantee diminution in energy consumption. To achieve energy efficiency, other power management with the aid of virtualisation should be jointly utilised, such as CPU throttling and dynamic reconfigurations that enable unused resources to be switched off.

However, initial implementations of virtualisation require all virtual nodes to use the same hardware platform. [86-88] present the idea of virtual machine migration that allows logical nodes to move among different hardware platforms without losing packets. In fact, the virtual machine migration that recovers the capacity and the features of Cloud systems can reduce the operational cost of the network system [89,90]. However, if this technique does not consider the limited memory capacity, limited processing power and limited communication bandwidth of hardware platforms, it would suffer from scalability problems for carrier-grade networks. Furthermore, migrating VMs from one node to another leads to energy overhead, because this process may require an important number of nodes to be powered on until the migration is complete. In [143], an interesting approach is proposed which could reroute traffic at layer 2, mainly by MPLS and Ethernet protocols instead of IP layer. The authors exploit the virtualization capabilities of layer 2 protocols in core networks, in which the router can transparently transfer all the virtual links from one (less used) to another putting the first one enter into standby mode. However, this solution adds a new part in the control plane, and needs further reconfigurations in order to maintain connectivity of physical topology.

The virtualisation technique, especially in MAN/WAN (or in any large infrastructures) needs more flexible management mechanisms to help from migrating and resuming virtual machines. It can also be coupled with traffic engineering capabilities to provide opportunities for foundations for energy-efficient and acceptable operation required by carrier-grade networks.

\section{c) Traffic engineering: with traditional protocols}

In networking terms, traffic engineering is a method that puts the traffic where the bandwidth is, by dynamic analysis of data traffic aimed at optimising the performance of telecommunication networks. The Energy-aware Traffic Engineering (ETE) strategy refers to routing traffic smartly based on energy-saving objectives. This aim is reached by bringing the network power consumption closely proportional to the actual traffic. A typical example of energy-aware traffic engineering consists of modifying the network protocols in order to route traffic over energy efficient paths, and switching off unused links. Typically in the ETE problem, the network is modelled as a graph, comprising a set of nodes interconnected by a set of unidirectional links. It is typically formulated as Mixed Integer Linear Programming (MILP), and the problem is known to be NP-hard. As a consequence, various heuristics are proposed. Several ETE approaches have been proposed by carefully handling the energy profile of network devices and the network routing [144-147]. The ETE may have the same taxonomy as the traffic engineering systems. It is well known that the traffic engineering systems can be done either with centralised or distributed decision, while their computation can be performed either offline or online.

In [91], an ETE scheme is proposed for carrier-Ethernet networks using Multiple Spanning Tree Protocol (MSTP). This approach computes the best subset of spanning trees and the best mapping of the traffic demands to the spanning trees, in such a way that a part of network is forced to be switched off. The traffic flows are then routed over the network over paths defined by the spanning trees (each spanning tree is assigned to a VLAN - virtual local area network). This is achieved by defining an optimisation model aimed at both considering the minimisation of power consumption and the load-balancing, subject to a set of 
constraints of traffic engineering; and ensuring minimal network performance. Load-balancing aims to reduce the link over-utilisation when the traffic load is high. However, the solution of such models is expensive to compute for large networks. Moreover, heuristics are rare; when they exist their performances are incompletely evaluated.

In reference [148], an interesting approach is proposed, called GRiDA, which builds upon link-state based protocols a distributed ETE. The turn-off decision is based on the feedback of past decisions history and the current link load, as well as the penalty of infeasible node configuration. Accordingly, GRiDA time complexity scales linearly with the network size $\mathrm{N}$ (number of nodes) and exponentially with nodal degree d, i.e., $\mathrm{O}\left(\mathrm{d} 2^{d}+\mathrm{dN}\right)$. To avoid the complexity of frequent configuration, in their next proposal [149], the authors devise a distributed solution based only on current topology configuration and the knowledge of traffic load on links. In [150], the power consumption is reduced making use optimisation modelling for simultaneous routing and bandwidth allocation. The major benefit of this proposal is that it does not rely on the assumption that traffic matrix is known, because its cost prediction. Hence, the authors resolve the problem introducing the notion of valuation of transmission service by employing a user utility function.

An important concept of mathematical optimisation, is called $\Gamma-$ robustness [151] [152], which has been successfully applied for backbone networks [153-155]. The EAR $\Gamma$-robustness-based allows to handle uncertain data, such as traffic demand volume and redundant traffic to be eliminated.

An energy-aware management approach is proposed in [156], exploiting the possibility to turn off nodes and interfaces. In addition, this work provides efficient greedy heuristic with different sorting policies. Note that a crucial step of the greedy heuristic is the way elements are sorted. The algorithm sorts elements, i.e., nodes and links, candidate to turn off using one of the following criteria: (i) most-power (MP), where MP iteratively selects the element which has the highest power consumption; (ii)least-flow (LF), where LF iteratively selects the element with the smallest amount of traffic already routed through it; (iii) least-link (LL) where LL iteratively selects the nodes with small number of its incident links; (iv) random (R), the element is selected randomly. If line cards are connected by multiple physical cables that form one logical bundled link (or bundle of lightpaths). This technique of composite link, called link aggregation, is standardized by the IEEE 802.1AX [157]. In this respect, we found relevant works have sketched the problem of ETE and network design with bundled links by turning off single physical cables [158$161]$.

[92] proposes a heuristic algorithm, Green Load-balancing Algorithm (GLA), which is able to optimise load balancing and energy efficiency jointly based on existing ETE algorithms for backbone networks. The GLA optimises the
Interior Gateway Protocol (IGP) link weights of a network, using a genetic algorithm to find the link weights that influence the traffic distribution within the network. Figure 17 illustrates the operation of the GLA algorithm for a simple traffic matrix composed of only two traffic demands: 30 units from $\mathrm{C}$ to $\mathrm{D}$ and 75 units from $\mathrm{A}$ to $\mathrm{D}$. The link utilisation of $C \rightarrow D$ is $60 \%, A \rightarrow D$ is $75 \%$. Thus the maximum link utilisation in this scenario is $75 \%$. The leastflow policy is adopted to iterate through the link set. If the link $C \rightarrow D$ is turned off, its load is rerouted through the alternative path $\mathrm{C} \rightarrow \mathrm{A} \rightarrow \mathrm{D}$. Then the maximum link utilisation on $\mathrm{A} \rightarrow \mathrm{D}$ increases to $105 \%$. Therefore, this link cannot be turned off because of network overload. On the other hand, the link $A \rightarrow D$ can be turned off because the alternative path $A \rightarrow B \rightarrow D$ will produce a maximum link utilisation of $60 \%$. In this example, only one link (link A$>$ D) can be turned off.

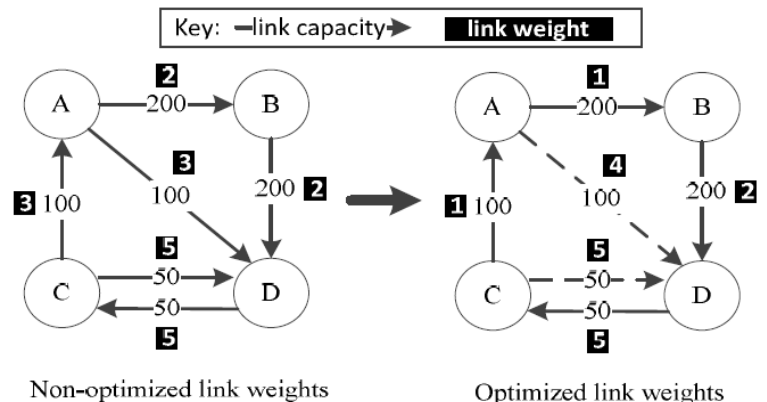

Figure 17. Example of the link weight optimisation adopted in [92]

If the link weights are optimised as shown on the right side of Figure 10, both links $C \rightarrow D$ and $A \rightarrow D$ can be turned off without affecting the network load. The traffic demands are then routed along the new paths $\mathrm{C} \rightarrow \mathrm{A} \rightarrow \mathrm{B} \rightarrow \mathrm{D}$ and $\mathrm{A} \rightarrow \mathrm{B} \rightarrow \mathrm{D}$ respectively. The resulting network ends up with maximum link utilisation of $52.5 \%$. Therefore, this algorithm can achieve simultaneous improvement of both energy saving and load balancing.

Regarding traffic engineering and load balancing performances, works [114] and [162] provide analytical models to capture the performance of energy-aware network devices, especially those compliant with ACPI standard, that include LPI and AR techniques. As well as, they trade off energy consumption devices for packet forwarding. Moreover, the optimisation policies presented in [162] focus on the packet processing with respect to the overall device consumption, and able to find the best way to distribute traffic among the packet processing engine's pipelines.

As the traffic matrices are considered independent, this algorithm can find different sets of link weights in the optimisation technique for each traffic matrix. A generic overview of ETE approach is presented in [93] and is shown in Figure 18. This approach uses the network topology, a power model of the network devices, and traffic matrix estimation (if offered) to pre-compute the sets of paths; three sets of paths are defined: always-on, on-demand, and failover. This approach defines a model that minimises the network energy consumption and puts the complementary set 
of links in power-saving mode. The decisions taken by the computation of the model are reassigned to the network routers to be installed in their routing tables. Traffic information is collected online and transferred to the processing functional blocks residing in the offline level. This approach allows traffic to be aggregated on the alwayson paths achieving low energy consumption at the low traffic levels, while an online component can progressively use the on-demand paths to satisfy the offered load.

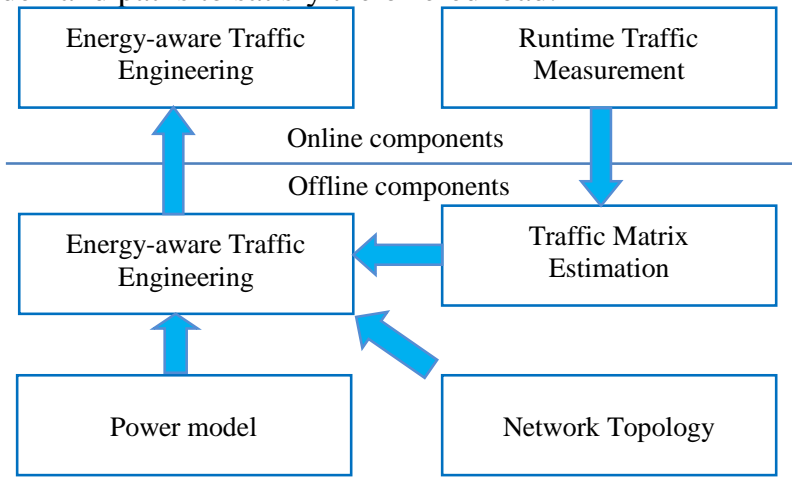

Figure 18. General overview of ETE[93]

Interesting works have dealt with the multi-period traffic optimisation; among others: [163-165, 190]. Two MILPbased algorithms are presented in [163], the first adopts a fixed configuration for routing along multi periods of daily time, while the second MILP allowing flexibility of routing path to the network administrator.

\section{d) Traffic engineering: Software defined network}

The Software Defined Network (SDN) is a novel networking paradigm showing significant promise through network programmability, and by splitting network functions between the forwarding elements and the controller element. Actually, SDN was introduced 20 years ago [94]. There are two main SDN technologies: OpenFlow [95] and ForCES; the second is called ProGFE (Programmable Generic Forwarding-Element) [96]. Recently, OpenFlow has succeeded in launching itself as an SDN industry engineering standard because it has less complex functionality compared to ProGFE. OpenFlow is the most widely used to support energy-aware functionalities [97]. It offers new prospects to deploy several energy-aware routing algorithms. Indeed, the Openflow architecture takes out the control software from the switches ("forwarding elements") and moves it to a chief part ("controller element") that is logically centralised; this enables non-negligible power saving in the switches at the cost of the power consumed by the controller.

Following green networking improvement, recent works have targeted both shortest path routing and ETE [99-101]. As the shortest path routing is supported in all OpenFlow controller platforms, it is powerful to use this protocol in order to enable energy efficiency in the network and to offer the shortest path routing.

ElasticTree [100] is one of the most popular approaches to ETE that utilises SDN in data center networks. It allows to a network admin running an energy-aware configuration which dynamically turn off not needed devices. The work in [166] proposes an ETE solution inside OpenFlow protocol, with the aid of the GAL (Green Abstraction Layer) [124]. This integration permits internal communication between network devices to interchange their power states. In this way, the OpenFlow controller becomes aware of the energy consumption of each network components. In [167], an extension of the work [166], is presented to include more power states based on link capacity instead of simple On-Off states. The technique takes as input, the topology, the logical resources and the traffic demands and finds a satisfying mapping, that minimise entire power consumption, between traffic demands and action and flow configuration.

Giroire et al. [98] propose an optimisation method to minimise the energy consumption in backbone networks subject to the rule space constraints of OpenFlow switch and general constraints reflecting the minimum of performance guarantees. To carry out an important variety of network applications, the flow table of an OpenFlow switch should hold a very large number of rules. However, this assumption is not fulfilled because, actually, the number of flow rules in the hardware switch is bounded by the TCAM (Ternary Content Addressable Memory) memory size. This kind of memory is very expensive and very power-hungry. This causes a major roadblock in large-scale OpenFlow deployments, particularly in energy-aware routing solutions whose main objective is to reduce the number of used links. Note that this problem is not limited to SDN, any ETE technique could face it. However, TCAM-based energyaware SDN issues received significant attention as shown in [168]. Figure 19 illustrates the limited rule space problem in energy-aware routing within the OpenFlow switch. Figure $12 \mathrm{~b}$ presents an optimal solution, since it uses a minimum number of active links and satisfies the capacity constraints. With limited rule space, the routing solution moves away from the optimal solution, as shown in Figure 19c. We notice that without rule space constraints, node 2 holds nine flows, whereas it cannot hold more than five in the case of the limited space rule. Only the second solution will be compatible with hardware whose capacity is limited to a number of flows less than nine.

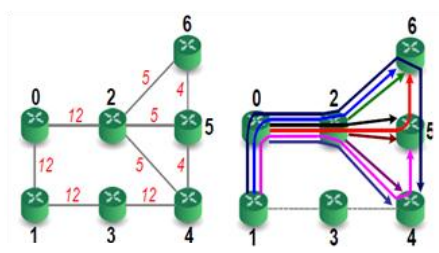

(a) Network and capacity on links (b) EAR unlimited rule space

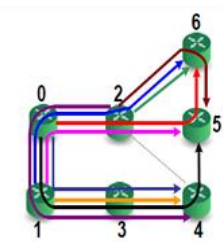

(c) EAR- limited
Figure 19. Limited rule space in OpenFlow switches [98]

By means of an exact formulation using the ILP model and greedy heuristic algorithm, the authors in [98] succeeded in computing the minimum number of links that must remain active, fulfilling the rule placement constraints. 
TABLE IX.

TAXONOMY OF UNDERTAKEN GREEN NETWORKING APPROACHES

\begin{tabular}{|c|c|c|c|c|c|}
\hline & & Proposal & Mechanisms & Targeted devices & Network layer \\
\hline \multirow{2}{*}{$\begin{array}{l}\overline{0} \\
\frac{0}{0} \\
\frac{0}{0} \\
z\end{array}$} & $\begin{array}{c}\text { Hardware } \\
\text { Optimization }\end{array}$ & $\begin{array}{c}{[22-24 ; 59-70 ; 133-140 ; 170-} \\
174]\end{array}$ & $\begin{array}{l}\text { Dynamic frequency and voltage of } \\
\text { processors }\end{array}$ & $\begin{array}{l}\text { Node chassis } \\
\text { LCs }\end{array}$ & $\begin{array}{l}\text { Data link } \\
\text { Multi-layer }\end{array}$ \\
\hline & $\begin{array}{c}\text { Software } \\
\text { Optimization } \\
\end{array}$ & {$[72-74]$} & Power management applications & Routers & Multi-layer \\
\hline \multirow{3}{*}{$\begin{array}{l}\overline{0} \\
\frac{0}{0} \\
\frac{y}{0} \\
0 \\
0 \\
0\end{array}$} & Proxying & {$[78,80,81,142]$} & Traffic analysis and sleeping & $\begin{array}{c}\text { Web servers } \\
\text { Routers } \\
\text { Switches }\end{array}$ & $\begin{array}{l}\text { Multi-layer } \\
\text { Application }\end{array}$ \\
\hline & Virtualisation & {$[82-84 ; 86-89,143]$} & $\begin{array}{c}\text { Virtual machines } \\
\text { Virtual machine migration }\end{array}$ & $\begin{array}{c}\text { Routers } \\
\text { Switches } \\
\text { LCs } \\
\end{array}$ & Application \\
\hline & $\begin{array}{c}\text { Traffic } \\
\text { Engineering }\end{array}$ & $\begin{array}{c}{[94-96,99-101,105,114,125,144-} \\
167,178-190,198]\end{array}$ & $\begin{array}{c}\text { MILP and heuristics } \\
\text { Math models and simulations }\end{array}$ & $\begin{array}{c}\text { Routers } \\
\text { Switches } \\
\text { LCs }\end{array}$ & $\begin{array}{l}\text { Network } \\
\text { Data link }\end{array}$ \\
\hline
\end{tabular}

Table IX summarises this section and reports the undertaken approaches of green networking with respect to the network layer applicable and the basic mechanisms. This section discussed practical approaches whose objective is to reduce the power consumed in wired networks in general. We described the main ideas belonging to these approaches and proposed to adopt those that fit especially carrier-grade networks. For instance, energy-aware traffic engineering fits traffic variation observed within carrier-grade networks. Indeed, network traffic is usually higher during the day and lower during the night, thus it is powerful to implement such a mechanism in order to put unused devices and/or redundant resources into the low power state. Virtualisation approaches are also another promising solution to enable energy saving in carrier-grade networks. In fact, with virtualisation, different services can share the same resources. Therefore, network operators do not need any more to dedicate hardware to each application or workload. In this section, we introduced and classified the fundamental techniques that could be used to green a carrier-grade network. The classification criterion comes from network technology deployment and its operation (i.e. at node level and network level).

\section{ISSUES IN CARRIER-GRADE NETWORKS}

This section summarises the major issues related to power saving in carrier-grade networks. Their requirements are resiliency, scalability, quality of service and service management.

At the moment, network operators try to improve the energy efficiency of their network and to provide the high service level required by their users. However, these two objectives are contentious: a trade-off becomes inevitable. From the state-of-the-art, performance metrics are energy efficiency metrics and QoS performance metrics. However, new metrics need to be defined for energy-efficient, resilient, and scalable networks; they are required to have a good evaluation of the satisfactory factor for carrier-grade networks.

Resiliency - The resilience of optical transport networks is a very important aspect (more so than general access networks). Indeed, a link of a core network supports, at each instance, thousands and sometime hundreds of thousands of user connections. Thus, a failure of one link may disrupt the communication service offer to a very large number of users. It is generally required for carrier-grade networks to resume service delivery within $50 \mathrm{~ms}$. To enable this reliability, core networks rely on redundant hardware components. Hence, the design of energy-efficient resilient networks has a significant impact on the resources required, and therefore on their power consumption. Network resilience can be categorised as protection or restoration [110]. Protection is a proactive mechanism, while restoration is a reactive mechanism. In protection mechanism, alternative paths are kept and reserved before a failure occurs. When a failure occurs, no further signalling is required to establish the protection paths. In the case of the restoration mechanism, the recovery paths are dynamically assigned, and resources are not reserved until failure occurs. When a failure occurs, extra signalling is required to establish the restoration path. Computing the number of deployed resources as well their capacities is a difficult tradeoff against power consumption. This tradeoff was considered in [111].

In order to trade-off the energy saving versus the resilience performance, the work of [99] proposes a flow restoration technique showing that is hard to achieve energy saving performing $50 \mathrm{~ms}$ maximum delay for failure recovery as required by carrier-grade network. Also a very recent study, [191], focuses on energy versus resiliency trade-off in optical 
inter-datacenter networks along with solutions on an elastic optical backbone. They consider two resilient designs MOPIC (Minimum Outage Probability in Cloud) [192] and RPMPC (Resilient Provisioning with Minimum Power Consumption) [193]. RPMPC shows a good compromising scheme to offer resilient provisioning with minimum energy consumption.

Scalability - Network scalability is vital to meet the constant demand growth. Providers require that the network scales to support hundreds of thousands of customers to address adequately metropolitan and regional supplied areas. The scalability requires a very large physical infrastructure that is interconnected through many switches and routers; it should allow users to reach a wide variety of applications. However, such an infrastructure brings high costs in terms of CAPEX and OPEX. Hence, reducing these costs presents a major challenge for the operators. In this regard, virtualisation is considered as a promising solution [100,111,112]. Also essential aspects have been investigated on how to minimise energy consumption for scalable networks, by optimising the internal architectures of optical and optoelectronic devices. For instance, [113] provide a detailed analysis of the scalability and energy efficiency requirements for board and chip level interconnects and the corresponding targets for the potential of optical technologies.

Only few works address energy saving versus scalability trade-off issues [194,195]. For instance, [194] introduces an OpenScale inter-data center architecture, which can be upgraded gradually from traditional electrical switching DCNs in a plug-and-play technology achieving lower operational cost.

Quality of Service - Nowadays, carrier-grade networks promise to offer a good quality of service to their end users. Moreover, energy saving is becoming a crucial concern. Good trade-off between these two potential opposing concerns is required. An analytical model in [114, 169] allows the impact of power-saving techniques (such as adaptive link and low power idle techniques) on the quality of service metrics to be characterized, as well as how they affect the overall system behavior. This model can initiate a large set of network switching architectures, technologies and components and is becoming an interesting estimation tool that can be effectively adopted inside optimisation techniques for the dynamic control of green networking equipment.

Service management- All services provided by network operators must be managed and supervised in order to ensure that they are operating as expected. The service provider must have the performance measurements to back up any service level claims. If a fault does occur, then the service provider has to identify which services have been impacted to react appropriately. When this functionality is combined with energy efficiency, specialised systems and equipment are required to be added into the network. For instance, to meet the service level agreements (SLAs) in optical networks, some strategies can be taken [116]: (1) physical impairment, energy efficiency techniques may augment the effect of physical impairments like the fibre loss or dispersion. [117] addresses this problem and shows that it can be solved by hosting design strategies that are both power- and impairment-aware; (2) differentiated quality of protection; [118] proposes an efficient policy to apply differentiated QoP by assigning different levels to demand with different SLA requirements. This application allows for a reduction of protection resources with significant energy efficiency; (3) lightpath preemption, based on the intuition that low-priority services can be provisioned with the possibility of being rejected when connected to a level of criticality must use network resources. In this regard, lightpath preemption levels are used to decide to which routes the optical signal must be assigned first, releasing the resources used by lightpaths with lower preemption levels.

\section{CONCLUSIONS}

This article has surveyed the state-of-the-art on energy consumption in the ICT sector and especially in carrier-grade networks. We have described the most suitable techniques that could improve the energy efficiency of carrier gradenetworks from two different aspects: the network and node level.

We have shown the key contributors to the power consumption of wired networks: network devices, network architecture and service scenarios. Since the device elements represent an important source of power consumption, we revised the energy consumption profiles for network devices. Indeed, the energy profiles of devices under various loads can influence the choice of energy-saving technique. Then, we discussed the key issues for building an energy-efficient network: green operation of devices, efficient network design, energy-aware routing, and renewable energy supplies.

After examining different approaches described in existing surveys, we proposed a new taxonomy of the energy-saving approaches based on two main categories. The classification criterion comes from network technology deployment and its operation (node level and network level).

Carrier-grade networks are energy-hungry infrastructures that run large-scale systems to deliver services. In this respect, we give useful references for workers who are interested in energy-efficient and wide networks. Future research should deal with the joint impact of the different approaches.

Aspects of carrier-grade networks, being resilience, scalability, and quality of service, make it more difficult to determine the best performance trade-off between energy efficiency and required functionalities. Although valuable solutions have been studied to save energy, to the best of our knowledge, there is no work that considers all the carriergrade requirements at the same time. Two key issues remain open: what is the best way to assess the trade-off between energy saving and network performance? And how to 
achieve a power consumption that is proportional to network load? These issues concern not only carrier-grade networks but also a large set of wired networks.

\section{REFERENCES}

[1] M. Lin, A. Wierman, L. L. H. Andrew, and E. Thereska. "Dynamic right-sizing for power proportional data centers", IEEE/ACM Transactions on Networking, vol. 21, no. 5 , pp. 1378-1391, 2013.

[2] J. M. Rabaey, M. Pedram, "Low power design methodologies", vol. 336, Springer Science \& Business Media, 2012.

[3] Global Energy Statistical Yearbook 2014. Addressing the Challenge of Energy Efficiency through Information and Communication Technologies. Technical Report. European Commission, European Communities, web site. [Online]. Available at: http://yearbook.enerdata.net/.

[4] R. Bolla, Bruschi, F. Davoli, F. Cucchietti, "Energy Efficiency in the Future Internet:A Survey of Existing Approaches and Trends in Energy-Aware Fixed Network Infrastructures", IEEE Communications Surveys \& Tutorials, vol. 13, no. 2, pp. 223-243, 2011.

[5] R. Bolla, R. Bruschi, A. Carrega, F. Davoli, D. Suino, "Cutting the energy bills of Internet Service Providers and telecoms through power management: An impact analysis", Computer Networks, vol. 56, no. 10, pp. 2320-2342, 2012.

[6] J. Baliga, R. Ayre, K. Hinton, R. Tucker, "Green Cloud Computing: Balancing Energy in Processing, Storage, and Transport" IEEE, vol. 99, no. 1, pp. 149-167, 2011.

[7] J. Mankoff, R. Kravets, E. Belvis, "Some Computer Science Issues in Creating a Sustainable World", IEEE Computer, vol. 41, no. 8, pp. $102-105,2008$

[8] J. Malmodin, P. Bergmark and D. Lundén, "The future carbon footprint of the ICT and E\&M sectors". Proceedings of the First International Conference on Information and Communication Technologies for Sustainability, ETH Zurich, 2013.

[9] J. Malmodin, P. Bergmark and D. Lundén, "Energy and carbon footprint performance metrics for the ICT sector based on LCA". Proceedings of the International Symposium on Sustainable Systems and Technologies (ISSN 2329-9169), 2014.

[10] British Telecom Group, "Sustainability Report 2009", http://www.btplc.com/Societyandenvironment/Ourapproach/Sustaina bilityreport/index.aspx.

[11] ITWales, "Green Evangelist to Call for Big Changes in Computer Use to Aid Environment" at ITWales Conf., 2007, http://www.itwales.com/997539.htm.

[12] Deutsche Telekom Group, "Ecological Indicators", http://crreport. telekom.de/site08/en/datenfakten/kennzahlen/oekologischekennzahlen-/index.php.

[13] W. D. Nordhaus, "To Slow or Not to Slow: the Economics of the Greenhouse Effect", The Economic Journal, vol. 101, pp. 920-937, 1991.

[14] M. Gupta and S. Singh, "Greening of the Internet," ACM SIGCOMM Conf. (SIGCOMM 03), Karlshue, Germany, pp. 19-26, 2003.

[15] O. Tamm, C. Hermsmeyer, A. Rush, "Eco-Sustainable System and Network Architectures for Future Transport Networks", Bell Labs Technical Journal, vol.14, no.4, p. 311, 2010.

[16] R. Tucker, "Green Optical Communications -- Part I: Energy Limitations in Transport", IEEE J. Sel. Top. Quantum Electron., vol. 17, no. 2, pp. 261-274, 2011.

[17] D. Collins, "Carrier grade voice over IP", ISBN. 0071363262, edition McGraw-Hill, 2003.

[18] P. Mahadevan, P. Sharma, S. Banerjee, and P. Ranganathan, "A power benchmarking framework for network devices", In Networking, Springer Berlin Heidelberg, pp. 795-808, 2009.
[19] L. Shang, L. S. Peh, and N. Jha, "PowerHerd: a distributed scheme for dynamically satisfying peak-power constraints in interconnection networks". IEEE Transactions on Computer-Aided Design of Integrated Circuits and Systems vol. 25, no. 1, pp. 92-110, 2006.

[20] J. Chabarek, J. Sommers, P. Barford, C. Estan, D. Tsiang, and S. Wright, "Power awareness in network design and routing", In IEEE International Conference on ComputerCommunications (INFOCOM), pp. 457-465, 2008.

[21] B. Florin. Cornea, A. Orgerie, L. Lefèvre, "Studying the Energy Consumption of data transfers in Clouds: the Ecofen approach", IEEE, International Conference on Cloud Networking (CloudNet), 2014

[22] C. Gunaratne, K. Christensen, B. Nordman, "Managing energy consumption costs in desktop PCs and LAN switches with proxying, split TCP connections, and scaling of link speed". International Journal of Network Management,vol. 15, no. 5, pp. 297-310. 2005.

[23] M. Allalouf, Y. Arbitman, M. Factor, R. Kat, K. Meth, D. Naor, "Storage modeling for power estimation". In ACM Israeli Experimental Systems Conference (SYSTOR), 2009.

[24] H. Dietz, W. Dieter, "Compiler and runtime support for predictive control of power and cooling". In Parallel and Distributed Processing Symposium (IPDPS), 2006.

[25] M. Wolf, "The Physics of Computing", ISBN: 9780128096161 , edition, Elsevier, 2017.

[26] US Department of Energy, "1.1.9 Buildings Share of U.S. Electricity Consumption," Buildings Energy Data Book, 2009 [Online], buildingsdatabook.eren.doe.gov/docs/xls_pdf/1.1.9.pdf.

[27] A. Bianzino, C. Chaudet, D. Rossi, J. Rougier, "A Survey of Green Networking Research", IEEE Communications Surveys \& Tutorials, vol. 14, no. 1, pp. 3-20, 2012.

[28] R. Bolla, F. Davoli, R. Bruschi, K. Christensen, F. Cucchietti, and S. Singh, "The potential impact of green technologies in next-generation wireline networks: Is there room for energy saving optimization?," IEEE Communications Magazine, vol. 49, no. 8, pp. 80-86, 2011.

[29] Y. Zhang, P. Chowdhury, M. Tornatore, and B. Mukherjee, "Energy efficiency in telecom optical networks", Communications Surveys \& Tutorials, IEEE, vol. 12,no. 4, pp. 441-458, 2010.

[30] R. Doverspike, K. K. Ramakrishnan, and C. Chase, "Structural overview of ISP networks," in Guide to Reliable Internet Services and Applications, Springer London, pp. 19-93, 2010.

[31] C. Lange, "Energy-related aspects in backbone networks," in Proceedings of 35th European Conference on Optical Communication (ECOC 2009), (Wien, AU), 2009.

[32] Metro Ethernet Forum, "Metro ethernet services - a technical overview", http://metroethernetforum.org/PDF_Documents/metroethernet services.pdf.

[33] C. Lange and A. Gladisch, "Energy consumption of telecommunication networks: a network operator's view", Workshop on Energy Footprint of ICT: Forecast and Network Solutions, San Diego, CA, 2009.

[34] J. Baliga, R. Ayre, W. V. Sorin, and R.S Tucker, "Energy consumption in access networks", IEEE Optical Fiber Communication/National Fiber Optic Engineers Conference (OFC/NFOEC), 2008.

[35] K. Hinton, J. Baliga, M. Feng, R. Ayre, R. S. Tucker, "Power consumption and energy efficiency in the Internet", Network IEEE, vol. 25, no. 2, pp. 6-12, 2011.

[36] A. Q. Lawey, T. E. El-Gorashi, and J. M. Elmirghani, "Distributed Energy Efficient Clouds Over Core Networks", IEEE Journal of Lightwave Technology, vol. 32, no. 7, pp. 1261-1281, 2014.

[37] A.C. Orgerie, M.D. Assuncao, and L. Lefevre, "A survey on techniques for improving the energy efficiency of large-scale distributed systems", ACM Computing Surveys (CSUR), vol. 46, no. 4, p. 47:1-47: 31, 2014. 
[38] Technology Roadmap "Energy-efficient Buildings: Heating and Cooling Equipment", International Energy Agency, 2011.

[39] J. Baliga, R. Ayre, K. Hinton, and R. S. Tucker, "Photonic Switching and the Energy Bottleneck", Proc. Internat. Conf. Photonics in Switching, San Francisco, CA, 2007.

[40] J. Baliga, R. Ayre, K. Hinton, W. Sorin, and R. Tucker, "Energy Consumption in Optical IP Networks", Journal of Lightwave Technology, vol. 27, no. 13, pp. 2391-2403, 2009.

[41] FP7 TREND (Towards Real Energy-efficient Network Design) Network of Excellence: http://www.fp7-trend.eu/.

[42] M. Meo, E. Lerouzic, R. Cuevas, C. Guerrero, "Research Challenges on Energy-Efficient Networking Design", Computer Communications, vol. 50, pp. 187-195, 2014.

[43] B. Wang, and S. Singh, "Computational Energy Cost Of TCP", In IEEE International Conference On Computer Communications (INFOCOM 2004), Twenty-third Annual Joint Conference of the IEEE Computer and Communications Societies vol. 2, pp. 206-216, 2004.

[44] L. Irish,, K. Christensen, "A Green TCP/IP to Reduce Electricity Consumed by Computers". In IEEE Southeast Conference, pp. 302305, 1998.

[45] A. Cianfrani, V. Eramo, M. Listanti, M. Marazza, and E. Vittorini, "An energy saving routing algorithm for a green OSPF protocol", In INFOCOM IEEE Conference on Computer Communications Workshops, pp. 1-5, 2010.

[46] W. Van Heddeghem, M. De Groote, W. Vereecken, D. Colle, M. Pickavet, P. De-meester, "Energy-effciency in telecommunications networks: Link-by-link versus end-to-end grooming". In Conference on Optical Network Design and Modeling (ONDM), 2010.

[47] I. Seoane, J. A. Hernandez, P. Reviriego, D. Larrabeiti, "Energyaware flow allocation algorithm for Energy Efficient Ethernet networks", pp. 1-5, IEEE SoftCOM, 2011.

[48] J. C. Cardona Restrepo, C. G. Gruber, C. Mas Machuca, "Energy Profile Aware Routing," Proc. Green Communications Workshop in conjunction with IEEE ICC'09, Germany, 2009.

[49] A. Qureshi, R. Weber, H. Balakrishnan, J. Guttag, and B. Maggs. Cutting the electric bill for internet-scale systems. In Proc. of SIGCOMM'09, 2009.

[50] "Directive 2009/28/EC of the european parliament and the council,"Article 16, Journal of the European Union, 2009.

[51] "Directive 2010/31/EC of the European Parliament and the council, Journal of the European Union, 2010

[52] "European Technology Platform for the Electricity Networks of the Future - Smartgrids," web site. [Online]. Available at: http://www.smartgrids.eu.

[53] J. Miller, L. Bird, J. Heeter, and B. Gorham, "Renewable Electricity Use by the U.S. Information and Communication Technology (ICT) Industry", National Renewable Energy Laboratory, 2015. [Online]. Available: www.nrel.gov/publications.

[54] T. T. Ye, "Analysis of power consumption on switch fabrics in network routers", ACM the 39th Annual Design Automation Conference, pp. 10-14, 2002.

[55] L. A. Barroso and U. Hölzle, "The Case for Energy-Proportional Computing," IEEE Computer, vol. 40, pp. 33-37, 2007.

[56] "IEEE Standard for Information Technology - Local and Metropolitan Area Networks - Specific requirements - Part 3: Carrier Sense Multiple Access with Collision Detection (CSMA/CD) Access Method and Physical Layer Specifications Amendment 5: Media Access Control Parameters, Physical Layers, and Management Parameters for Energy-Efficient Ethernet," IEEE Standard 802.3az2010 (Amendment to IEEE Std 802.3-2008), pp. 1-302, 2010.

[57] K. Christensen, P. Reviriego, B. Nordman, M. Bennett, Mehrgan Mostowfi, J. A. Maestro, I"EEE 802.3az: The Road to Energy Efficient Ethernet," IEEE Communication Magazine, vol. 48, no. 11, pp. 50-56, 2010.
[58] B. Zhai, D. Blaauw, et al. "Theoretical and Practical Limits of Dynamic Voltage Scaling," Design Automation Conference (DAC), 2004.

[59] A. Chen, "Berkeley Lab Researchers Are Developing EnergyEfficient Digital Network Technology," Research News Berkley Lab., March 2007. Available at: http://www.lbl.gov/ScienceArticles/Archive/EETDefficient- networks.html.

[60] A. Meyer, B. Nordman, "Buildings as Networks: Danger, Opportunity, and Guiding Principles for Energy Efficiency," Background Discussion, IEA Workshop on Digital Networks, Paris, France, 2007.

[61] University of Florida, "The Energy Efficient Internet project", funded by NSF and Cisco, 2012. http://www.csee.usf.edu/christen/ energy/main.html.

[62] G. Goth, "The Net's Going Green - Multipronged Approach Might Save Costs, Energy- and the Climate," IEEE Internet Computing, vol. 12, no.1, pp. 7-9, 2008.

[63] K. Christensen, B. Nordman, R. Brown, "Power Management in Networked Devices," IEEE Computer, vol. 37, no. 8, pp. 91-93, 2004.

[64] C. Gunaratne, K. Christensen, "Ethernet Adaptive Link Rate: System Design and Performance Evaluation," Proc. 31st IEEE Conf. Local Computer Networks (LCN 2006), Tampa, FL, USA, pp. 28-35, 2006.

[65] C. Gunaratne, K. Christensen, B. Nordman, and S. Suen. "Reducing the energy consumption of ethernet with adaptive link rate (ALR)". Computers, IEEE Transactions on, vol. 57, no. 4, pp. 448-461, 2008.

[66] C. Gunaratne, K. Christensen, and S. Suen, "Ethernet Adaptive Link Rate (ALR): Analysis Of A Buffer Threshold Policy", In IEEE Global Telecommunications Conference 1-6, 2006.

[67] M. Alonso, J.M. Martinez, V. Santonja, and P. Lopez, Reducing power consumption in interconnection networks by dynamically adjusting link width. In International European Conference on Parallel and Distributed Computing (Euro-Par). pp. 882-890, 2004.

[68] K. Sato, "Optical technologies that enable Green networks," 12th International Conference on Transparent Optical Networks (ICTON), pp. $1-4,2010$

[69] R. S. Tucker, "Green Optical Communications---Part II: IEEE Journal of Energy Limitations in Networks," Selected Topics in Quantum Electronics, no. 99, pp.1-14, 2010.

[70] M. Xia, M. Tornatore, Y. Zhang, P. Chowdhury, C. Martel, B. Mukherjee, "Greening the Optical Backbone Network: A Traffic Engineering Approach," IEEE International Conference on Communications (ICC), pp. 1-5, 2010.

[71] J. Baliga, R. Ayre, K. Hinton, and R.S Tucker, "Energy consumption in wired and wireless access networks," IEEE Communications Magazine, IEEE, vol. 49, no. 6, pp. 70-77, 2011.

[72] EcoInfo 2011. From Windows 95 to Windows 7. EcoInfo report.

[73] LessWatts 2010. Server power measurements. LessWatts report. [Online] Available at: https://software.intel.com/sites/default/files/LessWatts.orgwhitepaper.pdf

[74] The Advanced Configuration \& Power Interface (ACPI) Specification -Revision 4.0, http://www.acpi.info/.

[75] R. Buyya, C.S. Yeo, S. Venugopal, J. Broberg, and I. Brandic, "Cloud Computing and Emerging IT Platforms: Vision, Hype, and Reality for Delivering Computing as the 5th Utility," Future Generation Computer Systems, vol. 25, no. 6, pp. 599-616, 2009.

[76] V. Freeh, F. Pan, N. Kappiah, D. Lowenthal, and R. Springer, "Exploring the Energy-Time Tradeoff in MPI Programs on a PowerScalable Cluster". In IEEE International Parallel and Distributed Processing Symposium, (IPDPS), 2005.

[77] F. Blanquicet, and K. Christensen,. Managing energy use in a network with a new snmp power state mib. In 33rd IEEE Conference on Local Computer Networks (LCN 2008), pp. 509-511, 2008. 
[78] K. J. Christensen, F. B. Gulledge, "Enabling Power Management for Network-attached Computers", International Journal of Network Management, vol. 8, no.2, pp. 120-130, 1998.

[79] K. Sabhanatarajan and A. Gordon-Ross, "A Resource Efficient Content Inspection System for Next Generation Smart NICs," in Proceedings of the IEEE International Conference on Computer Design 2008. (ICCD 2008), (Lake Tahoe, California, USA), pp. 156163, 2008.

[80] Y. Agarwal, S. Savage, and R. Gupta. SleepServer: A software-only approach for reducing the energy consumption of PCs within enterprise environments. In USENIX Annual Technical Conference (ATC). USENIX Association, Berkeley, CA, USA, 2010.

[81] S. Nedevschi, J. Chandrashekar, J. Liu, B. Nordman, S. Ratnasamy, and N. Taft, "Skilled in the Art of Being Idle: Reducing Energy Waste in Networked Systems," in Proceedings of the 6th USENIX Symposium onNetworked Systems Design and Implementation (NSDI 2009), (Boston, Massachusetts, USA), 2009.

[82] S. Nanda and T.-C. Chiueh, "A Survey on Virtualization Technologies," Tech. Rep. TR179, Department of Computer Science, SUNY at Stony Brook, 2005.

[83] Cisco, "Router Virtualization in Service Providers", White paper, 2008 ,

http://www.cisco.com/c/en/us/solutions/collateral/routers/carrierrouting-system/white_paper_c11-512753.pdf

[84] R. Nathuji, and K. Schwan. VirtualPower: coordinated power management in virtualized enterprise systems. In ACM SIGOPS symposium on Operating systems principles (SOSP). pp. 265-278, 2007.

[85] A.-C. Orgerie, A, L. Lefevre, and J.-P. Gelas. Demystifying Energy Consumption in Grids and Clouds. In Work in Progress in Green Computing, IGCC Workshop, 2010.

[86] F. Travostino, P. Daspit, L. Gommans, C. Jog, C. de Laat, J. Mambretti, I. Monga, B. van Oudenaarde, S. Raghunath, and P. Y. Wang. Seamless live migration of virtual machines over the man/wan. Future Generation Computer Systems vol. 22, no. 8, pp. 901-907, 2006.

[87] Y. Wang, E. Keller, B. Biskeborn, J. van der Merwe, J. Rexford, "Virtual Routers on the Move: Live Router Migration as a NetworkManagement Primitive," Proc. ACM SIGCOMM, Seattle, WA, 2008.

[88] L. Grit, D. Irwin, A. Yumerefendi, and J. Chase. Virtual Machine Hosting for Networked Clusters: Building the Foundations for "Autonomic" Orchestration. In International Workshop on Virtualization Technology in Distributed Computing (VTDC), 2006.

[89] C. Clark, K. Fraser, S. Hand, J. G. Hansen, E. Jul, C. Limpach, I. Pratt, and A. Warfield. Live migration of virtual machines. In Symposium on Networked Systems Design \& Implementation (NSDI). pp. 273-286, 2005.

[90] Deliverable project: D2.1 End-user requirements, technology specifications and benchmarking methodologies, web site. [Online]. Available: https://www.econet-project.eu/Repository/Document/291.

[91] A. Capone, D. Corti, L.G. Gianoli, and B. Sans`o. "An optimization framework for the energy management of carrier ethernet networks with multiple spanning trees. Computer Networks", vol. 56, no. 17, pp. $3666-3681,2012$

[92] F. Francois, N. Wang, K. Moessner, S. Georgoulas, and K. Xu, "Green IGP link weights for energy-efficiency and load-balancing in IP backbone networks," In IEEE IFIP Networking Conference, pp. 1-9, 2013.

[93] N. Vasi, P. Bhurat, D. Novakovi, M. Canini, S. Shekhar, and D. Kosti,"Responsive, energy-proportional networks," EPFL, Tech. Rep., 2010.

[94] D. L. Tennenhouse and D. J. Wetherall, "Towards an active network architecture," SIGCOMM Comput. Commun. Rev., vol. 26, no. 2, pp. 5-17, 1996.

[95] N. McKeown, T. Anderson, H. Balakrishnan, G. M. Parulkar, L. L. Peterson, J. Rexford, S. Shenker, and J. S. Turner,"OpenFlow: enabling innovation in campus networks," Computer Communication Review, vol. 38, no. 2, pp. 69-74, 2008.

[96] R. Giladi and N. Yemini, "A programmable, generic forwarding element approach for dynamic network functionality," in Proc. PRESTO, pp. 19-24, 2009.

[97] D. Kreutz, F.M, Ramos, P. Esteves Verissimo, C. Esteve Rothenberg, S. Azodolmolky, and S. Uhlig, "Software-defined networking: A comprehensive survey," Proceedings of the IEEE, vol. 103, no. 1, pp. 14-76, 2015.

[98] F. Giroire, J. Moulierac, and T.K. Phan, "Optimizing rule placement in software-defined networks for energy-aware routing,". In IEEE Global Communications Conference (GLOBECOM), pp. 2523-2529, 2014.

[99] D. Staessens, S. Sharma, D. Colle, M. Pickavet, and P. Demeester, "Software Defined Networking: Meeting Carrier Grade Requirements," in 18th IEEE Workshop on Local Metropolitan Area Networks (LANMAN), pp. 1-6, 2011.

[100]B. Heller, S. Seetharaman, P. Mahadevan, Y. Yiakoumis, P. Sharma, S. Banerjee, and N. McKeown, "ElasticTree: Saving Energy in Data Center Networks,". In NSDI , vol. 10, pp. 249-264, 2010.

[101]N.H. Thanh, P.N. Nam, T.H Truong, N.T, Hung, L.K. Doanh, and R. Pries, "Enabling experiments for energy-efficient data center networks on OpenFlow-based platform". Fourth International Conference on IEEE Communications and Electronics (ICCE), pp. 239-244, 2012.

[102]A. Bianco, T. Bonald, D. Cuda, and R.-M. Indre, "Cost, power consumption and performance evaluation of metro networks," IEEE/OSA J. Opt. Comm. Netw., vol. 5, no. 1, pp. 81-91, 2013.

[103]F. Musumeci, D. Siracusa, G. Rizzelli, M. Tornatore, R. Fiandra, and A. Pattavina, "On the energy consumption of IP-over-WDM architectures". In International Conference Communications (ICC), pp. 3004-3008, 2012.

[104]B. Mukherjee, Optical WDM Networks, Springer Science \& Business Media, 2006

[105]G. Shen, S. K. Bose, T. H. Cheng, C. Lu, and T. Y. Chai, "Performance study on a WDM packet switch with limited-range wavelength converters," IEEE Communication Letters, vol. 5, no. 10 pp. 432-434, 2001.

[106]A. Autenrieth A. K. Ilwankar, C. M. Machuca, and J.P Elbers, "Power consumption analysis of opaque and transparent optical core networks". In 13th International Conference on Transparent Optical Networks, 2011.

[107]A. Tzanakaki, K. Katrinis, T. Politi, A. Stavdas, M. Pickavet, P. Van Daele, and P. Monti, "Dimensioning the future pan-European optical network with energy efficiency considerations". Journal of Optical Communications and Networking, vol. 3, no. 4, pp. 272-280, 2011.

[108]S. Verbrugge, D. Colle, M. Pickavet, P. Demeester, S. Pasqualini, A. Iselt, and M. Jäger, "Methodology and input availability parameters for calculating OpEx and CapEx costs for realistic network scenarios", Journal of Optical Networking, vol. 5, no. 6, pp. 509-520, 2006.

[109]D. Larrabeiti, P. Reviriego, J.A. Hernandez, et al. "Towards an energy efficient $10 \mathrm{~Gb} / \mathrm{s}$ optical ethernet: Performance analysis and viability, " Elsevier Optical Switching and Networking, vol. 8, no 3, pp. 131-138, 2011.

[110]J.-P. Vasseur, M. Pickavet, and P. Demeester, "Network recovery: protection and restoration of optical, SONET-SDH, IP and MPLS," Elsevier, 2004.

[111]X. Dong, T. E. El-Gorashi, J. M. Elmirghani, "On the Energy Efficiency of physical Topology Design for IP Over WDM Networks", vol. 30, no. 12, pp. 193-142, 2012.

[112]A. N. Udipi, N. Muralimanohar, R. Balasubramonian, "Towards Scalable, Energy-Efficient, Bus-Based On-Chip Networks", IEEE HPCA, pp. 1-12, 2010.

[113]D. Miller, "Device Requirements for Optical Interconnects to Silicon Chips", Proc. IEEE, vol.97, no.7, pp. 1166 -1185, 2009. 
[114]R. Bolla, R. Bruschi, A. Carrega, and F. Davoli, "Green network technologies and the art of trading-off," IEEE INFOCOM 2011 Green Communications and Networking Workshop (IEEE INFOCOM GCN), pp. $301-306,2011$.

[115] The Organisation for Economic Co-operation and Development (OECD), Nomura Research Institute (NRI) Presentation. [Online]. Available: http://www.oecd.org/sti/ind/45009540.pdf

[116]Y. Ye, FJ. Arribas, J. Elmirghani, F. Idzikowski, J.L. Vizcaíno, P. Monti, and W. Van Heddeghem, "Energy-efficient resilient optical networks: Challenges and trade-offs". IEEE Communications Magazine, vol. 53, no. 2, pp. 144-150, 2015.

[117]C. Cavdar, C., M. Ruiz, P. Monti, L. Velasco, and L. Wosinska, "Design of Green Optical Networks with Signal Quality Guarantee," Proc. IEEE ICC, Ottawa, Canada, 2012.

[118]J. Lo, Y. Ye, F. Jime, and P. M. Krummrich, "Energy- and CostEfficient Protection in Core Networks by a Differentiated Quality of Protection Scheme," Proc. ECOC, London, U.K., 2013.

[119]https://iet.jrc.ec.europa.eu/energyefficiency/sites/energyefficiency/file s/files/COC_Energy-

Consumption/code_of_conduct_broadband_equipment_v4_1_final.pd $\mathrm{f}$.

[120] Y. Suh, K. Kim, A. Kim, and Y. Shin, "A study on impact of wired access networks for green Internet". Journal of Network and Computer Applications, vol. 57, pp. 156-168, 2015.

[121]The Green Touch initiative, http://www2.alcatellucent. com/blog/2010/01/ green-touch-making-communicationsnetworks 1000-times-more-energy-efficient/.

[122] http://www.econet-project.eu.

[123]R. Bolla, R. Bruschi, F. Davoli, F. Cucchietti, "Setting the course for a green Internet", Science, Letter to the Editor, vol. 342, no. 6164, p. 1316, 2013.

[124]R. Bolla, R. Bruschi, F. Davoli, L. Di Gregorio, P. Donadio, L. Fialho, M. Collier, A. Lombardo, D. Reforgiato Recupero, T. Szemethy, "The Green Abstraction Layer: A standard power management interface for next-generation network devices", IEEE Internet Computing, vol. 17, no. 2, pp. 82-86, March-April 2013.

[125]R. Bolla, R. Bruschi, F. Davoli, P. Donadio, L. Fialho, M. Collier, A. Lombardo, D. Reforgiato, V. Riccobene, T. Szemethy, "A northbound interface for power management in next generation network devices", IEEE Communications Magazine, vol. 52, no. 1, pp. 149-157, Jan. 2014.

[126]Eman Status Pages, https://tools.ietf.org/wg/eman/. B. Schoening, M. Chandramouli, B. Nordman, "Energy Management (EMAN) Applicability Statement" (No. RFC 7603), 2015.

[127]J. Parello, B. Claise, B. Schoening, and J. Quittek, "Energy management framework "(No. RFC 7326), 2014.

[128]F. A. Moghaddam, P. Lago, P. Grosso, "Energy-Efficient Networking Solutions in Cloud-Based Environments: A Systematic Literature Review", ACM computing Surveys, vol. 47, no. 4, art. 64, 2015.

[129]B. Kitchenham, O. P. Brereton, D. Budgen, M. Turner, J. Bailey, and S. Linkman, "Systematic literature reviews in software engineering-a systematic literature review". Information and software technology, vol. 51, no. 1, pp. 7-15, 2009.

[130]B. Addis, A. Capone, G. Carello, L. G. Gianoli, and B. Sansò, "Energy management in communication networks: a journey through modelling and optimization glasses". Computer Communications, vol. 91, pp. 76-94, 2016.

[131]C. Gunaratne, K. Christensen, and B. Nordman, "Managing Energy Consumption Costs in Desktop PCs and LAN Switches with Proxying, Split TCP Connections, and Scaling of Link Speed," Int'1 J. Network Management, vol. 15, no. 5, pp. 297-310, Sept./Oct. 2005.

[132]P. Reviriego, J. A. Hernadez, D. Larrabeiti, and J. A. Maestro, "Burst transmission for energy-efficient ethernet". IEEE Internet Computing, vol. 14 , no. 4 , pp. 50-57, 2010.
[133]A. Chatzipapas, and V. Mancuso, "Modelling and real-trace-based evaluation of static and dynamic coalescing for energy efficient ethernet". In Proceedings of the fourth international conference on Future energy systems, ACM, pp. 161-172, 2013.

[134]S. Herreria-Alonso, M. Rodriguez-Perez, M. Fernandez-Veiga, C. Lopez-Garcia, "A Power Saving Model for Burst Transmission in Energy-Efficient Ethernet,” IEEE Commun. Lett., vol. 15, no. 5, pp. 584-586, 2011.

[135]S. Herreria-Alonso, M. Rodriguez-Perez, M. Fernandez-Veiga, C. Lopez-Garcia," "A GI/G/1 Model for $10 \mathrm{~Gb} / \mathrm{s}$ Energy Efficient Ethernet Links," IEEE Trans. Commun., vol. 60, no. 11, pp. 33863395, 2012.

[136]M. Ajmone Marsan, A. F. Anta, V. Mancuso, B. Rengarajan, P. R. Vasallo, G. Rizzo, "A Simple Analytical Model for Energy Efficient Ethernet,” IEEE Commun. Lett., vol. 15, no. 7, pp. 773-775, 2011.

[137]P. Reviriego, J. A. Hernandez, D. Larrabeiti, J. A. Maestro, "Performance Evaluation of Energy Efficient Ethernet," IEEE Commun. Lett., vol. 13, no. 9, pp. 697-699, 2009.

[138]P. Reviriego, K. Christensen, J. Rabanillo, J. Maestro, "An Initial Evaluation of Energy Efficient Ethernet," IEEE Commun. Lett., vol. 15, no. 5, pp. 578-580, 2011.

[139]R. Bolla, R. Bruschi, A. Carrega, F. Davoli, P. Lago, "A closed-form model for the IEEE 802.3az network and power performance", IEEE Journal on Selected Areas in Communications, vol. 32, no. 1, pp. $16-$ 27, 2014.

[140] R. Bolla, R. Bruschi, A. Carrega, and F. Davoli, "An analytical model for designing and controlling new-generation green devices", IEEE Globecom Workshops, pp. 1388-1393. 2010.

[141]"ProxZZZy for sleeping hosts," Standard ECMA-393, June 2012. [Online]. Available: http://www.ecma-international.org/publications/ files/ECMA-ST/ECMA-393.pdf

[142]R. Bolla, R. Khan, M. Repetto, "Assessing the Potential for Saving Energy by Impersonating Idle Networked Devices," IEEE Journal on Selected Areas in Communications, vol. 34 no. 5, pp. 1676-1689, 2016

[143]R. Bolla, R. Bruschi, A. Cianfrani, M. Listanti, "Enabling backbone networks to sleep," IEEE Network, vol. 25, no. 2, pp. 26-31, 2011.

[144]M. Aicardi, R. Bruschi, F. Davoli, P. Lago, "A decentralized team routing strategy among telecom operators in an energy-aware network", Proc. SIAM Conf. on Control and Its Applications, pp. 340-347, 2015.

[145]R. Bolla, R. Bruschi, P. Lago, "Energy adaptation in multi-core software routers," Computer Networks, vol. 65, pp. 111-128, 2014.

[146]A. Vishwanath, K. Hinton, R. Ayre, R. Tucker, "Modelling Energy Consumption in High-Capacity Routers and Switches", IEEE Journal on Selected Areas in Communications, vol. 32, no. 8, 2014.

[147]R. G. Garroppo, S. Giordano, G. Nencioni, M. G. Scutellà, "PowerAware Routing and Network Design with Bundled Links: Solutions and Analysis," Journal of Computer Networks and Communications vol. 2013, Article ID 154953, 2013.

[148]A. P. Bianzino, L. Chiaraviglio, M. Mellia, J.-L. Rougier, "GRiDA: Green Distributed Algorithm for Energy-Efficient IP Backbone Networks" Computer Networks, vol. 56, no.14, pp. 3219-3232, 2012.

[149]A. P. Bianzino, L. Chiaraviglio, and M. Mellia, "Distributed algorithms for green IP networks," in INFOCOM Workshops, pp. 121-12, 2012.

[150]P. Jaskóła, P. Arabas, A. Karbowski, "Simultaneous Routing and Flow Rate Optimization in Energy-Aware Computer Networks," Int. J. Appl. Math. Comput. Sci., vol. 26, no. 1, pp. 231-243, 2016.

[151] D. Bertsimas, M. Sim, "Robust discrete optimization and network flows, Mathematical Programming", vol. 98, no. 1-3, pp. 49-71, 2003.

[152]D. Bertsimas, M. Sim, The price of robustness, Operations Research 52 (1) (2004) 35 - 53. doi:10.1287/opre.1030.0065. 
[153]D. Coudert, A. Kodjo, T. K. Phan, "Robust energy-aware routing with redundancy elimination," Computers \& Operations Research, vol. 64, pp. 71-85, Dec. 2015.

[154]S. Duhovnikov, A. Koster, M. Kutschka, F. Rambach, and D. Schupke, " $\Gamma$-robust network design for mixed-line-rate-planning of optical networks". In National Fiber Optic Engineers Conference (pp. JTh2A-02). Optical Society of America, 2013.

[155]B. Addis, A. Capone, G. Carello, L.G. Gianoli, and B. Sans`o. A robust optimization approach for energy-aware routing in mpls networks. In 2013 International Conference on Computing, Networking and Communications (ICNC), pages 567-572. IEEE, January 2013.

[156]L. Chiaraviglio, M. Mellia, F. Neri, "Minimizing ISP Network Energy Cost: Formulation and Solutions," IEEE/ACM Transactions on Networking, vol. 20, no. 2, pp. 463-476, April 2012.

[157]IEEE Standard 802.1 AX: Link Aggregation, 2008.

[158]R. G. Garroppo, S. Giordano, G. Nencioni, M. G. Scutellà, "Mixed Integer Non-Linear Programming models for Green Network Design," Computers and Operations Research, vol. 40, no. 1, pp. 273281, Jan. 2013.

[159]Garroppo, R. G., Giordano, S., Nencioni, G., \& Scutellà, M. G. (2013). Power-aware routing and network design with bundled links: Solutions and analysis. Journal of Computer Networks and Communications, 2013.

[160]G. Lin, S. Soh, and K. W. Chin, "Energy-aware traffic engineering with reliability constraint". Computer Communications, vol. 57, pp. 115-128, 2015.

[161]R. G. Garroppo, S. Giordano, G. Nencioni, M. Pagano, and M. G. Scutell'a, "Energy saving heuristics in backbone networks," in Proc. SustainIT, Pisa, Italy, Oct. 2012.

[162]R. Bolla, R. Bruschi, A. Carrega, F. Davoli, "Green networking with packet processing engines: Modeling and optimization", IEEE/ACM Transactions on Networking, vol. 22, no. 1, pp. 110-123, 2014.

[163]B. Addis, A. Capone, G. Carello, L. G. Gianoli, B. Sansò, "Energy Management Through Optimized Routing and Device Powering for Greener Communication Networks," IEEE/ACM Transactions on Networking, vol. 22, no. 1, pp. 313-325, 2014.

[164]F. Francois, K. Moessner, and S. Georgoulas. Optimizing link sleeping reconfigurations in isp networks with off-peak time failure protection. IEEE Transactions on Network and Service Management, 10(2):176-188, 2013.

[165]J. Moulierac, and T. K. Phan, "Optimizing IGP link weights for energy-efficiency in multi-period traffic matrices". Computer Communications, vol. 61, pp. 79-89, 2015.

[166]R. Bolla, R. Bruschi, F. Davoli, C. Lombardo, A. Lombardo, G. Morabito, V. Riccobene, "Green extension of OpenFlow", in Proc. ITC 26 Workshop on Energy-Efficiency, Programmability, Flexibility and Integration in Future Network Architectures (EPFI), Karlskrona, Sweden, 2014.

[167]R. Bolla, R. Bruschi, F. Davoli, C. Lombardo, "Fine-grained energyefficient consolidation in SDN networks and devices", IEEE Transactions on Network and Service Management, vol. 12, no. 2, pp. 132-145, 2015.

[168] M. F. Tuysuz, Z. K. Ankarali, and D. Gözüpek, "A Survey on Energy Efficiency in Software Defined Networks". Computer Networks, 2016.

[169]R. Bolla, R. Bruschi, A. Carrega, F. Davoli, "Green networking with packet processing engines: Modeling and optimization", IEEE/ACM Transactions on Networking, vol. 22, no. 1, pp. 110-123, Feb. 2014.

[170]R. Bruschi, F. Davoli, M. Mongelli, "Adaptive frequency control of packet processing engines in telecommunication networks", IEEE Communications Letters, vol. 18, no. 7, pp. 1135-1138, 2014.

[171]R. Bolla, R. Bruschi, F. Davoli, P. Lago, Á. Bakay, R. Grosso, M. Kamola, M. Karpowicz, L. Koch, D. Levi, G. Parladori, D. Suino, "Large-scale validation and benchmarking of a network of powerconservative systems using ETSI's Green Abstraction Layer",
Transactions on Emerging Telecommunications Technologies, vol. 27, no. 3, pp. 451-468, 2016.

[172]E. Niewiadomska-Szynkiewicz, A. Sikora, P. Arabas, M. Kamola, M. Mincer, J. Kołodziej, "Dynamic power management in energy-aware computer networks and data intensive computing systems", Future Generation Computer Systems, vol. 37, pp. 284-296, 2014.

[173]E. Niewiadomska-Szynkiewicz, A. Sikora, P. Arabas, J. Kołodziej, "Control system for reducing energy consumption in backbone computer networks", Concurrency and Computation: Practice and Experience, vol. 25, no. 12, pp. 1738-1754, 2013.

[174]L. Chiaraviglio, A. Cianfrani, E. Le Rouzic, M. Polverini, "Sleep Modes Effectiveness in Backbone Networks with Limited Configurations," Computer Networks, vol. 57, no. 15, pp. 2931-2948, 2013.

[175]F. Idzikowski, S. Orlowski, C. Raack, H. Woesner, A. Wolisz, "Dynamic Routing at Different Layers in IP-over-WDM NetworksMaximizing Energy Savings," Optical Switching and Networking, vol. 8, no. 3, pp. 181-200, 2011.

[176]G. Shen, R. Tucker, "Energy-Minimized Design for IP over WDM Networks," IEEE/OSA Journal of Optical. Communications and Networking, vol. 1, no. 1, pp. 176-186, 2009.

[177]X. Dong, T. El-gorashi, J. M. H. Elmirghani, "On the Energy Efficiency of Physical Topology Design for IP over WDM Networks," IEEE Journal of Lightwave Technology, vol. 30, no. 12, pp. 1931-1942, 2012.

[178]B. Wu, S. Fu, X. Jiang, H. Wen, “Joint Scheduling and Routing for QoS Guaranteed Packet Transmission in Energy Efficient Reconfigurable WDM Mesh Networks," IEEE Journal on Selected Areas in Communications, vol. 32, no. 8, pp. 1533-1541, 2014.

[179]B. Birand, H. Wang, K. Bergman, D. Kilper, T. Nandagopal, G. Zussman, "Real-Time Power Control for Dynamic Optical Networks - Algorithms and Experimentation," IEEE Journal on Selected Areas in Communications, vol. 32, no. 8, pp. 1615-1628, 2014.

[180]F. Musumeci, M. Tornatore, A. Pattavina, “A Power Consumption Analysis for IP-over-WDM Core Network Architectures," IEEE/OSA Journal of Optical Communications and Networking, vol. 4, no. 2, pp. 108-117, 2012.

[181]Y. Zhang, M. Tornatore, P. Chowdhury, B. Mukherjee, "Energy Optimization in IP-over-WDM Networks," Optical Switching and Networking, vol. 8 no. 3, pp. 171-180, 2011.

[182]J. Zhang, M. T. Hosseinabadi, N. Ansari, "Standards-Compliant EPON Sleep Control for Energy Efficiency: Design and Analysis," IEEE/OSA Journal of Optical Communications and Networking, vol. 5, no. 7, pp. 677-685, 2013.

[183]I. Cerutti, N. Sambo, P. Castoldi, "Sleeping Link Selection for Energy-Efficient GMPLS Networks," IEEE Journal of Lightwave Technology, vol. 29, no. 15, pp. 2292-2298, 2011.

[184]A. Coiro, M. Listanti, A. Valenti, F. Matera, "Reducing Power Consumption in Wavelength Routed Networks by Selective Switch Off of Optical Links," IEEE Journal of Selected Topics in Quantum Electronics, vol. 17, no. 2, pp. 428-436, 2011.

[185]N. H. Bao, L. M. Li, H. F. Yu, Z. Z. Zhang, H. B. Luo, "PowerAware Provisioning Strategy with Shared Path Protection in Optical WDM Networks," Optical Fiber Technology, vol. 18, no. 2, pp. 81$87,2012$.

[186]F. Musumeci, M. Tornatore, J. Lopez Vizcaino, Y. Ye, A. Pattavina, "Energy-Efficiency of Protected IP-over-WDM Networks with SleepMode Devices," Journal of High Speed Networks, vol. 19, no. 1, pp. 19-32, 2013.

[187]J. Kani, S. Shimazu, N. Yoshimoto, H. Hadama, "Energy-Efficient Optical Access Networks: Issues and Technologies," IEEE Communications Magazine, vol. 51, no. 2, pp. S22-S26, 2013.

[188]J. I. Kani, "Power Saving Techniques and Mechanisms for Optical Access Networks Systems," IEEE/OSA Journal of Lightwave Technology, vol. 31 no.4, pp. 563-570, 2013.

[189]L. Valcarenghi, D. P. Van, P. G. Raponi, P. Castoldi, D. R. Campelo, S. Wong, S. Yamashita, "Energy Efficiency in Passive Optical 
Networks: Where, When, and How?," IEEE Network, vol. 26 no. 6 , pp. 61-68, 2012.

[190]E. Amaldi, A. Capone, L. G. Gianoli, "Energy-aware IP traffic engineering with shortest path routing," Computer Networks, vol. 57, no. 6, pp. 1503-1517, 2013.

[191]Z. I. Rauen, B. Kantarci, and H. T. Mouftah, "Resiliency versus energy sustainability in optical inter-datacenter networks", Optical Switching and Networking, vol. 23, pp. 144-155, 2017.

[192]B. Kantarci, and H. T. Mouftah, "Resilient optical inter-data-center network design", In Transparent Optical Networks (ICTON), pp. 1-4, 2014.

[193]B. Kantarci, and H. T. Mouftah, "Minimum outage probability provisioning in an energy-efficient cloud backbone", In Global Communications Conference (GLOBECOM), pp. 2879-2884, 2013.

[194]Zhang, D., Guo, H., Yang, T., \& Wu, J. (2017). Optical Switching based Small-world Data Center Network. Computer Communications.

[195]Mellette, W., Schuster, G., Porter, G., Papen, G., \& Ford, J. (2016). A Scalable, Partially Configurable Optical Switch for Data Center Networks. Journal of Lightwave Technology.

[196] Global e-Sustainability Initiative (GeSI), "SMARTer2020: The Role of ICT in Driving a Sustainable Future", Report; [online] http://gesi.org/SMARTer2020.

[197] even Global e-Sustainability Initiative (GeSI), \#SMARTer2030 ICT Solutions for 21st Century Challenges, 2015; [online] http://smarter2030.gesi.org).

[198]R. Maaloul, L. Chaari, and B. Cousin, Energy-aware forwarding strategy for Metro Ethernet networks, in Computer Systems and Applications Conference (AICCSA), pp. 1-7, 2015, 